\title{
The sedimentation of colloidal nanoparticles in solution and its study using quantitative digital photography
}

\author{
Johanna Midelet ${ }^{1}$, Afaf H. El-Sagheer ${ }^{2}$, Tom Brown ${ }^{2}$, \\ Antonios G. Kanaras ${ }^{1}$, and Martinus H. V. Werts*3 \\ ${ }^{1}$ University of Southampton, Physics and Astronomy, Faculty \\ of Physical Sciences and Engineering, Southampton SO171BJ, \\ U.K. \\ ${ }^{2}$ University of Oxford, Department of Chemistry, 12 Mansfield \\ Road, Oxford, OX1 3TA, U.K. \\ ${ }^{3}$ Ecole normale supérieure de Rennes, CNRS, lab. SATIE, \\ Campus de Ker Lann, F-35170 Bruz, France
}

author version, accepted manuscript published as

Part. Part. Syst. Charact. 2017, 1700095

DOI: $10.1002 / p p s c 201700095$

\begin{abstract}
Sedimentation and diffusion are important aspects of the behaviour of colloidal nanoparticles in solution, and merit attention during the synthesis, characterisation and application of nanoparticles. Here we study the sedimentation of nanoparticles quantitatively using digital photography and a simple model based on the Mason-Weaver equation. Good agreement between experimental time-lapse photography and numerical solutions of the model was found for a series of gold nanoparticles. The new method was extended to study for the first time the gravitational sedimentation of DNA-linked gold nanoparticle dimers as a model system of a higher complexity structure. Additionally we derive simple formulas for estimating suitable parameters for the preparative centrifugation of nanoparticle solutions.
\end{abstract}

*martinus.werts@ens-rennes.fr 


\section{Introduction}

Observations on the sedimentation of colloidal solutions were important historically in establishing the physical reality of molecules and providing a molecular basis for thermodynamics in the form of statistical mechanics.[13] Nowadays, there is an intense interest in the development of colloidal solutions of engineered nanocrystals for a variety of applications. These solutions should display sedimentation behaviour in line with expectations for these particles on basis of their shape, composition and suspending medium.

For stable and dilute solutions of sufficiently large particles, the sedimentation behaviour (Figure 1) is a result of the particular interplay of nanoparticle hydrodynamics, and gravitational and Brownian forces. Under these conditions, the surface chemistry of the nanoparticles, while ensuring colloidal stability, does not significantly influence the sedimentation behaviour. For smaller particles $(<10 \mathrm{~nm})$, and in particular 'soft' particles such as polymers and proteins, solvation effects on sedimentation may become significant, as the shell of solvent molecules around the object influences its hydrodynamic behaviour.[4-6] Furthermore, the presence of surfactants interacting with colloidal particles may change sedimentation behaviour[7, 8]. These effects are not considered in the present work.

A general understanding of the sedimentation of nanoparticle solutions is useful as it may give rapid and visual clues about the size distribution and colloidal stability of newly synthesised colloids. These clues go beyond the simple assessment of whether a prepared solution is colloidally stable. Careful monitoring of sedimentation behaviour may be used to verify the nature of the suspended object in its native medium, and is complementary to methods that characterise a limited number of specimens deposited on a substrate, such as electron microsopy. Furthermore, sedimentation, decantation and controlled centrifugation are often purification steps in wetchemical synthesis of nanoparticles and nanoparticle assemblies, for instance for the purification of gold nanorods.[9] A further example is the purification of nanoparticles by flocculation and subsequent sedimentation. Flocculation under the influence of depletion forces due to specific flocculatants produces large aggregates of these nanoparticles that settle rapidly leaving non-flocculated impurities in the supernatant.[10-12].

When analysing the interaction of nanoparticles with biological systems in therapeutic and diagnostic applications, their transport properties such as diffusion and sedimentation need to be taken into account The importance of sedimentation to in vitro studies of cellular uptake of nanoparticles has been demonstrated.[13]

There is a clear connection between the gravitational sedimentation of 


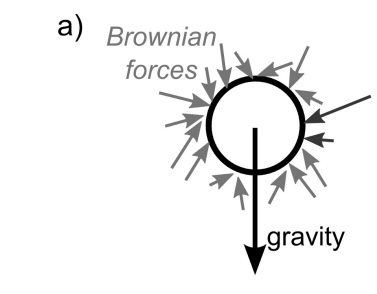

b)
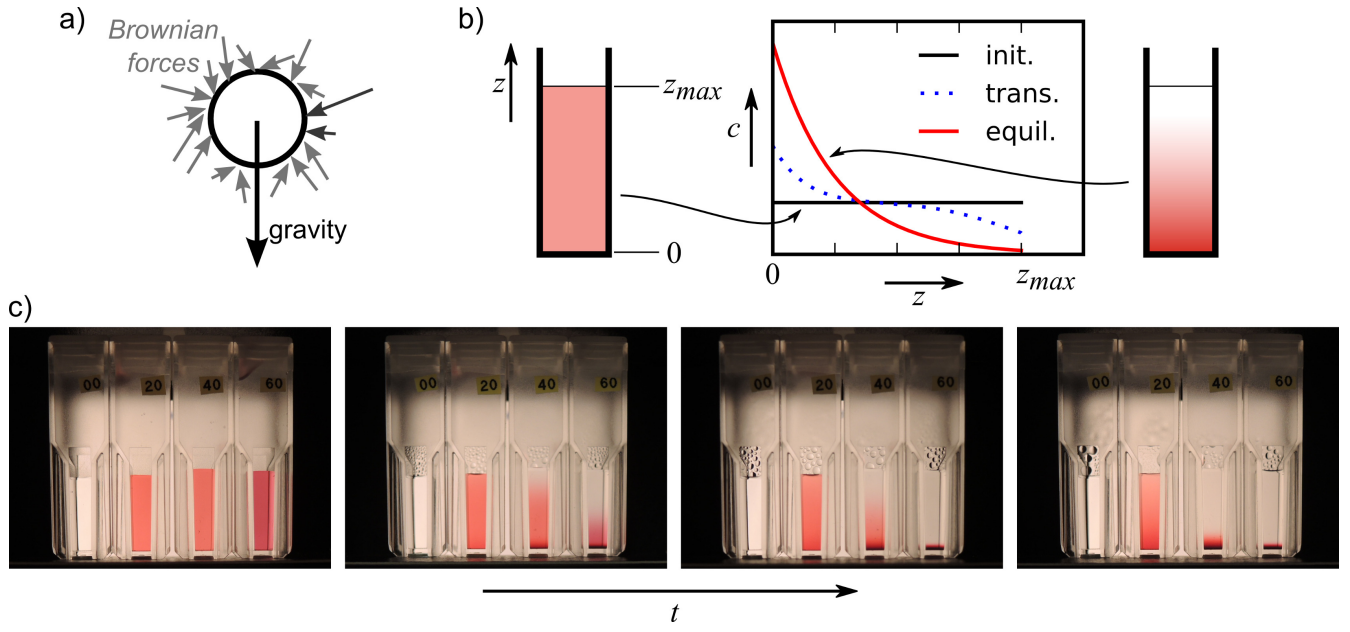

Figure 1 - Sedimentation versus Brownian diffusion in colloidal solutions. (a) Gravity tends to direct dense particles to the bottom of the cell, whereas the random Brownian forces tend to disperse the particles throughout the entire volume. (b) This leads to the establishment of an equilibrium gradient starting from an initial homogeneous distribution of particles. Here we trace the theoretical initial, transient and equilibrium concentration profiles as a function of vertical position. (c) Experimental observation of sedimentation over time of colloidal gold $(20,40,60 \mathrm{~nm})$ in water using quantitative digital photography. The cell at the left of each picture contains water only. Photos were taken at the start, and after 7, 14 and 35 days, respectively. The rightmost picture represents the cells at equilibrium: a photograph of the Boltzmann distribution.

colloidal particles and analytical centrifugation techniques, where sedimentation is sped up by increasing $g$ to multiples of the earth gravitational pull. Analytical ultracentrifugation (AUC), which is well known for its applications in biochemistry, has been successfully applied to the study of nanoparticle solutions.[14-17] Optimisation of the measurement using a spectroscopic approach in combination with detailed numerical analysis of the multi-wavelength sedimentation profiles has made AUC extremely versatile for the analysis of nanoparticle preparations.[18] It has furthermore been demonstrated that information on the shapes of DNA-based nanoparticle assemblies can also be obtained for AUC sedimentation analysis combined with hydrodynamic modeling.[19]

The rotational speed and centrifugal acceleration in analytical ultracentrifuges are extremely high, and adapted to proteins and small bioparticles. As will be further pointed out later on, many assemblies of inorganic nanoparticles, which have higher density and size, are better analysed with 
more modest speeds. A relevant recent development in this respect is analytical centrifugation (AC) with space- and time-resolved extinction profiles (STEP), [20, 21] which functions at significantly lower speeds than AUC. Its application to nanoparticle solution analysis is currently emerging.[22, 23] Another analytical centrifuge technique is differential centrifugal sedimentation (DCS) which is based on the sedimentation of injected samples in a spinning disk containing a liquid. DCS successfully detects small changes in particle sedimentation behaviour as a result of their ligand capping, [24] or particle size variations due to varying synthesis conditions.[25]

For these reasons, it is of fundamental interest and instructive to study the sedimentation behaviour of nanoparticle solutions in greater detail. Recently, Alexander et al. reported a study of gravitational sedimentation of gold nanoparticles [26]. They focused on measuring the optical density at a pre-defined height in the solution as a function of time using an UV-visible absorption spectrophotometer. This study was then further extended by making estimates of the size distribution of colloidal gold samples by including a multi-wavelength analysis.[27]. Sedimentation has also been instrumental for in situ attenuated total internal reflection infrared spectroscopy by concentrating the particles or their aggregates onto the substrate.[28]. Magnetic-field enhanced sedimentation of superparamagnetic iron oxide particles has also been investigated.[29]

Here we take advantage of quantitative digital photography[30-32] for experimentally analyzing the time-evolution of the concentration gradient of sedimenting nanoparticle solutions. The technique is simple and easily implemented. When properly set up, time-lapse imaging of the entire nanoparticle concentration gradient is possible (Figure 1c). In particular with nanoparticles of high overall density (such as those having a gold core) sedimentation may be readily studied without a centrifuge, using only the gravitational field.

The aim of the present work was to monitor the nanoparticle sedimentation process and the establishment of a concentration gradient using quantitative digital photography, and model this with a simple mathematical diffusion-sedimentation equation. We also discuss the practical implications of the theoretical model for the analysis and purification of nanoparticle assemblies. Furthermore we obtain a photograph of the Boltzmann distribution (rightmost picture of Figure 1c). This work utilises well-known concepts from colloid chemistry with an emphasis on their practical application in purification and characterization of colloidal nanoparticle assemblies. 


\section{Sedimentation vs diffusion: the Mason-Weaver equation}

A simple model for sedimentation can be formulated under the assumptions that there are no interactions between nanoparticles and that their motion is only governed by random Brownian forces and a directional gravitational force. The relevant parameters of the system depend only on the effective density and overall shape of the particles, and on the density, viscosity and temperature of the suspending liquid. The surface chemistry of the particles has no influence, nor does the exact composition of the suspending medium. However, these chemical parameters may influence the model indirectly, e.g. by changing density or viscosity of the medium, or by altering particle shape and effective density. We work under conditions where liquid density and viscosity are not significantly altered by the dissolved ingredients, and at low densities of colloid (mass fraction $\ll 1 \%$ ).

The establishment of a concentration gradient in such a dilute colloidal solution of independent, non-interacting particles in a homogeneous gravitational field is described by the Mason-Weaver equation, [33] a one-dimensional partial differential equation. This equation is the direct predecessor to the more well-known Lamm equation[34, 35] which applies for a centrifugal field and is extensively used in the analysis of ultracentrifuge data.[36]

The height position in the cell is given by $z$. The concentration of decanting particles $c$ satisfies the Mason-Weaver equation (1), with boundary conditions (2).

$$
\begin{gathered}
\frac{\partial c}{\partial t}=D \frac{\partial^{2} c}{\partial z^{2}}+s g \frac{\partial c}{\partial z} \\
D \frac{\partial c}{\partial z}+s g c=0\left(z=z_{\max }, z=0\right)
\end{gathered}
$$

$D$ is the diffusion coefficient, $s$ sedimentation coefficient, $g$ gravitational constant, and $z=z_{\max }$ and $z=0$, are the top and bottom of the cell; respectively. In this work, equations (1) and (2) are solved numerically using a Crank-Nicolson finite-difference method which is described in the Supporting Information, Section S.2. The numerical solver accepts an arbitrary concentration profile as the initial condition.

The initial condition at $t=0$ that we are interested in is $c=c_{0}$ for $0 \leq z<z_{\max }$ and $c=0$ elsewhere. That is, initially the particles are homogeneously distributed within the cell.

The term $s g$ is the terminal velocity of a particle accelerated by gravitational force in a viscous liquid $\left(v_{\text {term }}=s g\right.$ ), and represents the velocity 
(directed towards the bottom of the cell) this particle would have in absence of Brownian motion. It is written in terms of the sedimentation coefficient $s$. The sedimentation coefficient $s$ for a specific type of particle in a particular viscous liquid is

$$
s=\frac{m_{b}}{f}
$$

This involves the buoyant mass $m_{b}$ (i.e. the mass of the particle minus the mass of the liquid displaced by the particle) and the frictional coefficient $f$. The frictional coefficient depends on the particle's size and shape and on the viscosity of the solvent (more on this below). The frictional coefficient $f$ also is of importance for the diffusion coefficient $D$.

$$
D=\frac{k_{\mathrm{B}} T}{f}
$$

For spherical particles we have the well-known results from Stokes' law and the Einstein-Smolukowski-Sutherland theory given by equations (5) and (6).

$$
f_{\text {sphere }}=6 \pi \eta a \quad m_{b, \text { sphere }}=\frac{4}{3} \pi a^{3}\left(\rho-\rho_{\mathrm{fl}}\right)
$$

where $a$ is the particle radius, $\eta$ the viscosity of the liquid, and $\rho$ and $\rho_{\mathrm{fl}}$ the mass density of the particle and the suspending fluid, respectively. An underlying assumption of these relations is the non-slip condition at the sphere-liquid interface.

$$
s_{\text {sphere }}=\frac{m_{b}}{6 \pi \eta a}=\frac{2}{9} \frac{a^{2}\left(\rho-\rho_{\mathrm{fl}}\right)}{\eta} \quad D_{\text {sphere }}=\frac{k_{\mathrm{B}} T}{6 \pi \eta a}
$$

Table 1 contains diffusion and sedimentation coefficients for gold nanospheres calculated using Eqns. (6) for selected diameters in water at $277 \mathrm{~K}$, which is the temperature used in the experiments (see Experimental Details). Data for these particles at $298 \mathrm{~K}$ can be found in the Supporting Information, Tables S2, S3, S4 and S5. The table collects further characteristic values related to sedimentation, which will be discussed below.

At longer times, the solution to the Mason-Weaver equation converges to the concentration distribution at equilibrium, eqn. (7).

$$
c(z)=B c_{0} \exp \left(\frac{-z}{z_{0}}\right)
$$

The characteristic height of the equilibrium gradient, $z_{0}$, is 
Table 1 - Calculated sedimentation parameters and characteristic values for gold nanospheres in water at $277 \mathrm{~K}\left(4^{\circ} \mathrm{C}, \eta=1.56 \mathrm{mPa} . \mathrm{s}\right)$. Diffusion $(D)$ and sedimentation $(s)$ coefficients, characteristic height of concentration gradient at equilibrium $\left(z_{0}\right)$, approximate time to equilibrium from completely dispersed state $\left(t_{\text {sed }}^{1 \mathrm{~cm}}\right)$, concentration factor $\left(B^{1 \mathrm{~cm}}\right)$. The two latter values refer to a liquid column height of $1 \mathrm{~cm}$. Further data at $298 \mathrm{~K}$ can be found in the Supporting Information.

\begin{tabular}{lccrcc}
\hline $\begin{array}{l}\text { diam. } \\
\mathrm{nm}\end{array}$ & $\begin{array}{c}D \\
\mu \mathrm{m}^{2} \mathrm{~s}^{-1}\end{array}$ & $\begin{array}{c}s \\
10^{-9} \mathrm{~s}\end{array}$ & $\begin{array}{r}z_{0} \\
\mathrm{~mm}\end{array}$ & $\begin{array}{c}t_{\text {sed }}^{1 \mathrm{~cm}} \\
\text { hours }\end{array}$ & $B^{1 \mathrm{~cm}}$ \\
\hline 13 & 19.9 & 0.110 & 18.5 & 2579 & 1.29 \\
20 & 13.0 & 0.260 & 5.08 & 1090 & 2.29 \\
40 & 6.48 & 1.04 & 0.636 & 272 & 15.7 \\
50 & 5.18 & 1.62 & 0.325 & 174 & 30.7 \\
60 & 4.32 & 2.34 & 0.188 & 121 & 53.1 \\
80 & 3.24 & 4.16 & 0.0794 & 68.1 & 126 \\
100 & 2.59 & 6.50 & 0.0407 & 43.5 & 246 \\
150 & 1.73 & 14.6 & 0.0121 & 19.4 & 830 \\
\hline
\end{tabular}

$$
z_{0}=\frac{D}{s g}
$$

Interestingly, by combining (3), (4) and (8), we find that

$$
z_{0}=\frac{k_{\mathrm{B}} T}{m_{b} g}
$$

The same result for the equilibrium gradient can be obtained using a statistical mechanical approach, i.e. calculating the Boltzmann distribution for dense suspended particles in a gravitational field $\left(E_{\text {particle }}=m_{b} g z\right)$, illustrating that the digital image of the gold nanoparticle solution at equilibrium is a photograph of a Boltzmann distribution. The concentration profile at equilibrium does not depend on the frictional coefficient nor on the viscosity of the medium, but exclusively on the buoyant mass of the particles. For a spherical particle this translates into

$$
z_{0, \text { sphere }}=\frac{3 k_{\mathrm{B}} T}{4 \pi a^{3}\left(\rho-\rho_{\mathrm{fl}}\right) g}
$$

Some typical values for the characteristic height $z_{0}$ for gold nanospheres in water are included in Table 1. For diameters from 13 to $60 \mathrm{~nm}$, the 
characteristic height falls in the 20 to $0.2 \mathrm{~mm}$ range, where the gradient is readily observed with the naked eye.

Going from the initial dispersed solution to the equilibrium gradient leads to an increase in concentration of the particles near the bottom of the cell. In Eqn. (7) this is expressed in the $B$ constant, which represents the factor by which the particles are concentrated at the bottom. It is obtained by applying mass conservation to Eqn. (7). With the initial condition being a uniform concentration $c_{0}$ throughout the cell $\left(0 \leq z<z_{\max }\right)$, we obtain eqn. (11). Typical values for gold nanosphere have been added to Table 1.

$$
B=\frac{z_{\max }}{z_{0}\left[1-\exp \left(-z_{\max } / z_{0}\right)\right]}
$$

We found that the time to reach sedimentation equilibrium from the initial homegeneously dispersed state can be roughly estimated from the time $t_{\text {sed }}$ necessary for a hypothetical particle to traverse the cell entirely from top to bottom (distance $z_{\max }$ ) at its sedimentation velocity $s g$.

$$
t_{\mathrm{sed}}=\frac{z_{\mathrm{max}}}{s g}
$$

Comparison of the numerical solutions for various ratios of $z_{\max }$ to $s g$ to the corresponding equilibrium distributions given by Eqn. (7) showed that the concentration gradient at $t_{\text {equil }} \approx 1.4 t_{\text {sed }}$ is in all cases within less than one percent of the final equilibrium distribution (see Figure S3). This is a useful result which defines the necessary time window for sedimentation experiments and simulations. Weaver[37] found analytically that the upper limit for $t_{\text {equil }}$ is $2 t_{\text {sed }}$,

A more sophisticated analysis of the time to reach equilibrium is known in the literature.[38] In the Supporting Information we demonstrate that this analysis validates $t_{\text {equil }} \approx 1.4 t_{\text {sed }}$ as a rough guess (which is already a 30 percent time reduction with respect to the Weaver limit), but in many cases the time needed to reach equilibrium is actually shorter still, and for optimisation of experimental procedures the more elaborate formulation can be helpful. Moreover, the numerical solver described in the present work enables detailed simulation of the evolution of the concentration profile, and may be even more complete for optimising the sedimentation and detection strategy.

In $1 \mathrm{~cm}$ high liquid columns, the time needed to reach equilibrium vary from hours to weeks for typical gold nanospheres (Table 1). In practice, with the data analysis demonstrated in this paper, it will not be necessary to run experiments to complete equilibrium, as good estimates for diffusion 
and sedimentation coefficients can be extracted using the initial progression of the concentration gradient.

\section{Sedimentation of simple gold nanospheres}

Experimental concentration profiles of several settling solutions of gold nanoparticles were obtained from digital photographs taken at different moments. A typical example, using $40 \mathrm{~nm}$ gold nanospheres in water, is shown in Figure 2. The photographs for this series (of which 4 are shown in Figure 1) were taken over a 39 day period, and quantitative vertical optical density profiles were obtained using the method detailed in the Experimental section. In this work, we only use the green colour channel of the images, since this produces the strongest optical response for the red-coloured gold nanoparticles.

In the same figure we also show the solution of the Mason-Weaver equation $c(z, t)$ at the same times $t$. The diffusion and sedimentation coefficients were adjusted independently to obtain best agreement with the experimental observations. The values obtained, $D=5.1 \times 10^{-12} \mathrm{~m}^{2} \mathrm{~s}^{-1}$ and $s=7.9 \times 10^{-10} \mathrm{~s}$, agree within $20 \%$ with those expected from the EinsteinSmolukowski-Sutherland and the Stokes relations for perfect $40 \mathrm{~nm}$ diameter gold spheres in water at $277 \mathrm{~K}$ (Table 1).

Diffusion and sedimentation coefficients were obtained for the entire series of gold nanosphere diameters by fitting the Mason-Weaver solutions to the optical density profiles evolving in time. These coefficients agree reasonably well (within 20\%) with the predicted values (Figure 3). In addition to the predictions based on the gold core only, we also calculated the expected diffusion coefficients for the gold core plus an extra $1 \mathrm{~nm}$ of ligand shell ( $\rho=900 \mathrm{~kg} \mathrm{~m}^{-1}$, dotted curve in Fig. 3). This second theoretical curve demonstrates that the present simple method can not distinguish between small differences in overall hydrodynamic radius and density.

These experiments on simple gold nanosphere solutions demonstrate the feasibility of quantitative sedimentation measurements using digital photography. The solutions behave according to the Mason-Weaver model. It is also illustrated that equilibrium gradients develop for gold nanospheres in the selected diameter range upon standing undisturbed for prolonged times. These are observable to the naked eye.

The simplicity of the method and its implementation obviously leads to a variety of sources of experimental error. The cumulation of these errors finally leads to an overall error in the diffusion and sedimentation coefficients that is estimated above to be around $20 \%$, based on the comparison of the experimental results with the idealized theoretical curves (Figure 3). 

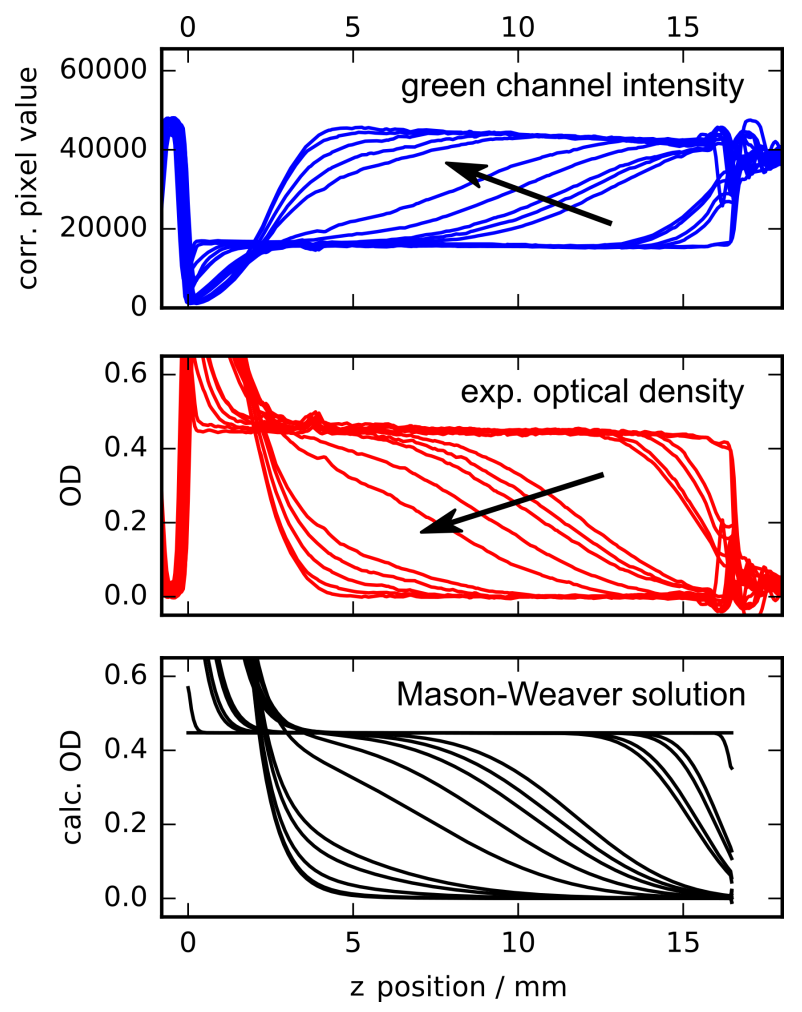

Figure 2 - Evolution of the vertical particle density gradient in an aqueous solution of $40 \mathrm{~nm}$ diameter gold nanospheres towards sedimentation equilibrium, observed using quantitative digital photography at $277 \mathrm{~K}$. Photos were taken at $t=0,1 \mathrm{~h}, 21 \mathrm{~h}, 27 \mathrm{~h}, 44 \mathrm{~h}, 52 \mathrm{~h}, 7 \mathrm{~d}, 9 \mathrm{~d}, 11 \mathrm{~d}, 14 \mathrm{~d}, 21 \mathrm{~d}$, $23 \mathrm{~d}, 28 \mathrm{~d}, 35 \mathrm{~d}$, and 39 days. Top: line-averaged intensities in the green colour channel, corrected for differences in illumination intensity between different images. Middle: experimental optical density profiles obtained from the intensity profiles. The arrow indicates the direction of time. Bottom: theoretical optical density profiles obtained numerically as the solution to the Mason-Weaver equation, with $D=5.1 \times 10^{-12} \mathrm{~m}^{2} \mathrm{~s}^{-1}$ and $s=7.9 \times 10^{-10} \mathrm{~s}$. 
The back-illumination of the observation cells should be homogeneous, which is not entirely the case in our set-up. The small illumination inhomogeneities are corrected for by the baseline correction we apply. Small deviations in camera positioning from photograph to photograph lead to changes in optical path lengths across the spectroscopic cells. There are also fluctuations in illumination intensity; these are small and averaged out when quantitatively analyzing the time-lapse series using the Mason-Weaver model, but lead to additional noise. Stray light is present in the optical system, i.e. the dark zones of the image are not entirely devoid of light, which limits the maximum optical density that can be reliably measured. A specific optical design with dedicated illumination and detection optics may improve the analysis of the concentration gradient.

The long duration of the sedimentation in some samples calls for efficient sealing of the containers, since solvent evaporation reduces the liquid column height, introducing further uncertaintity in the analysis. Also, the the meniscus at the top of the liquid column and the optical artefacts (dark zone) related to it limit the precision of the measurement of the gradient.

At present, the numerical analysis assumes an ideally monodisperse distribution of nanoparticles, all being of the same shape and volume. The diffusion and sedimentation coefficients obtained are effective, average coefficients. We have not attempted to analyse the data in terms of continuous size distributions, in which case an additional weighting should be applied for the strong dependence of the optical extinction coefficient on the diameter of gold nanoparticles.[39]

There is no fundamental impediment to analyzing the time-lapse optical extinction profile data sets using models based on continuous polydisperse size distributions, or even multimodal distributions, similarly to what is currently already done in analytical ultracentrifugation. $[15,20,36]$ The numerical solver presented here may be used to generate time-resolved concentration profiles for individual nanoparticle diameters in a trial distribution. After weighting with the effective extinction coefficients these can then be combined into the expected overall optical extinction profiles, which can then be optimised to fit the experimental data. In this respect, multimodal size distributions may be challenging if the different populations display unsufficient contrast in diffusion and sedimentation coefficients. 

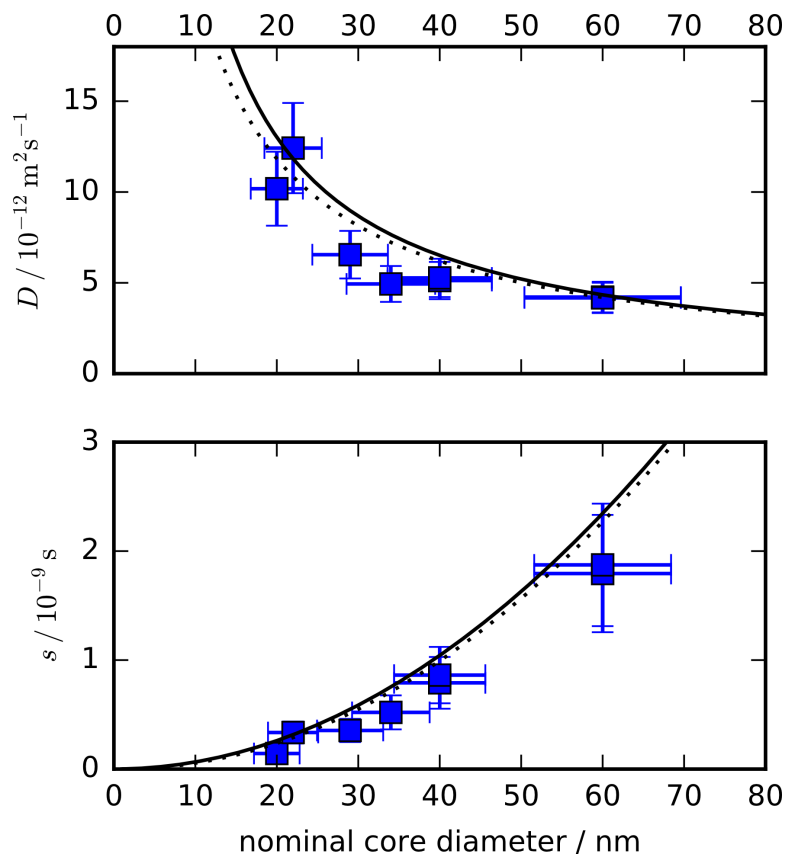

Figure 3 - Diffusion $D$ and sedimentation $s$ coefficients obtained by analysing the evolving experimental density gradient of settling gold nanosphere solutions (square markers). The solid curves are the expected values for perfect golden spheres from the Stokes-Einstein-Sutherland equation (top), and Stokes' law (bottom). The dotted curves are for gold spheres with a hypothetical $1 \mathrm{~nm}$ thick organic layer. 


\section{Application to nanoparticle assemblies}

After the initial demonstration of the quantitative analysis of time-lapse photography of settling spherical gold nanoparticles in water, we investigated a sample of purified DNA-linked dimers of $13 \mathrm{~nm}$ diameter gold nanospheres (Figure 4). This experiment illustrates that studying sedimentation can aid in the chemistry and characterization of biomolecularly-scaffolded multinanoparticle assemblies.

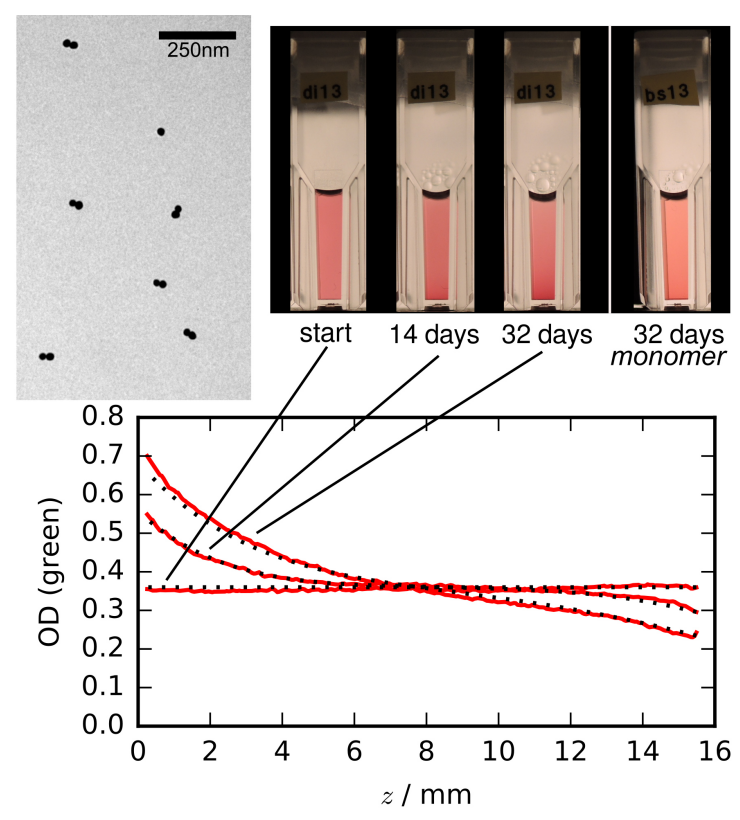

Figure 4 - Top left: transmission electron micrograph of purified DNA-linked dimer sample. Top right: composed image of the time-lapse photography of sedimenting DNA-linked gold nanosphere dimers (at $277 \mathrm{~K}$ ). The rightmost cell is a sample of monomeric $13 \mathrm{~nm}$ gold nanospheres. Bottom: optical density traces as a function of vertical position in the cell, taken at various points in time (red solid lines); the black dotted curves are the solution to the MasonWeaver equation with $D=5.8 \times 10^{-12} \mathrm{~m}^{2} \mathrm{~s}^{-1}$ and $s=9.0 \times 10^{-11} \mathrm{~s}$

By adjusting the curves to the experimental data, we obtain $D=5.8 \times$ $10^{-12} \mathrm{~m}^{2} \mathrm{~s}^{-1}$ and $s=9.0 \times 10^{-11} \mathrm{~s}$, for the diffusion and sedimentation coefficients, respectively. The sedimentation coefficient of DNA-linked dimers is slightly smaller than that of a bare $13 \mathrm{~nm}$ monomer sphere, i.e. it sediments even more slowly. The extra mass from the second gold sphere is counterbalanced by more friction with the solvent due to the larger outer surface area of the dimer. The larger volume comes to a large extent from the DNA 
which has a much lower density than gold.

Additionally, the diffusion coefficient is lower than that of a $13 \mathrm{~nm}$ monomer as a result of the larger hydrodynamic volume of the object. For the characteristic height (Eqn. 3) we find $z_{0}=6.6 \mathrm{~mm}$. This value is close to what we would expect for an object which simply has the double buoyant mass of a $13 \mathrm{~nm}$ monomer. Since gold has a much higher density than water, whereas the DNA linker has a density comparatively very close to that of water. Any extra volume taken up by the DNA linkage does not significantly contribute to the buoyant mass of the object, since the displaced water volume is replaced with a substance having a density close to that of water. The buoyant mass of the dimer is therefore determined by the two gold cores. On the other hand, the bulky DNA structure should indeed have an effect on the sedimentation and diffusion constants, i.e. sedimentation will be greatly slowed down compared to an equivalent sphere of the same buoyant mass.

The lower sedimentation coefficients leads to slower establishment of the gradient. However, in combination with the lower diffusion coefficient, it finally leads to a more pronounced, shorter density gradient. A further analysis of the hydrodynamic behaviour and the resulting combination of $D$ and $s$ of this type of assemblies is not within the scope of this work, but has received recent attention in the literature.[19]

It is also interesting to note that the estimated time to obtain the final equilibrium gradient (at 277K, with $2 \mathrm{~cm}$ liquid height) is on the order of 200 days for both the monomers and the DNA-linked dimers. For analysing the transient concentration profile it is not necessary to wait that long, which demonstrates the interest of having the numerical solution to the MasonWeaver at hand. Nonetheless, 30 days is still a long time, and these samples are better analysed with centrifuge-based techniques.[14, 16, 19, 24, 25, 40]. In this context, it is interesting to note that - as we will see in the following

- the required centrifugal acceleration for many inorganic-core nanoparticles is well within range of standard laboratory centrifuges, instead of higherspeed specialised instruments.

\section{Centrifugation of nanoparticle solutions}

The results from the Mason-Weaver model may be used to generate intial approximate estimates for preparative and analytical centrifugation. For many nano-assemblies using high-density inorganic core materials, the centrifugal acceleration necessary for rapid sedimentation is well within the capabilities of standard table-top laboratory centrifuges. A centrifugal acceleration that is much higher than strictly necessary may have deleterious consequences 
for colloidal stability as the density of nanoparticles in the pellet may become very high, accelerating aggregation. This is one reason why even a rough estimate is of interest. Furthermore, we anticipate that preparative purification protocols may be developed for nanoparticle assemblies by using approximate numerical simulations, using the computer code supplied with the present work.

As an example we consider the minimal centrifugal acceleration $g_{\text {cfg }}$ needed to obtain sedimentation equilibrium for gold colloid solutions in a centrifuge within a given time $t_{\mathrm{cfg}}$, i.e. we wish to establish the centrifugal conditions for a typical nanoparticle 'centrifugation/re-dispersal' washing procedure. In Section 2 of this paper, we found that the time to reach equilibrium is $1.4 t_{\text {sed }}$, and using Eqn. (12) we can roughly estimate the centrifugal acceleration $g_{\mathrm{cfg}}$ necessary for the chosen centrifugation time $t_{\mathrm{cfg}}$ and a liquid height in the centrifuge tube $z_{\text {tube }}$.

$$
g_{\mathrm{cfg}}=\frac{1.4 \times z_{\text {tube }}}{s \times t_{\mathrm{cfg}}}
$$

This can be expressed as 'relative centrifugal force' ('times $g$ ', RCF = $g_{\mathrm{cfg}} / g$, where $\left.g=9.81 \mathrm{~m} \mathrm{~s}^{-1}\right)$.

$$
\mathrm{RCF}=\frac{1.4 \times z_{\text {tube }}}{g \times s \times t_{\mathrm{cfg}}}
$$

As discussed above and detailed in the Supporting Information, the factor 1.4 represents a conservative rough estimate of the time to reach equilibrium. This factor may become smaller as a function of the particle's sedimentation and diffusion coefficients and the container height, using the formula by Van Holde and Baldwin,[38] or using results from numerical simulation. However, for the sake of simplicity, we will use 1.4 for the following calculations, as this is a robust albeit conservative choice.

Table 2 contains the calculated RCF values needed for complete centrifugation of gold colloids in typical Eppendorf-type vials (liquid height $z_{\text {tube }}=3 \mathrm{~cm}$ ), within $t_{\mathrm{cfg}}=30 \mathrm{~min}$. These values were then used in an experiment in which a selection of colloidal gold solutions were spun for 30 min. at the calculated RCF. The samples were subjected to a typical centrifugation/re-dispersal cycle, in which $95 \mathrm{vol} \%$ of the supernatant was removed, followed by redispersal of the (relatively fluid, but highly concentrated) pellet in fresh water. In most cases the recommended centrifugal acceleration gave remarkably good results (Supporting Information, Figure S7).

Measurement of the UV-visible extinction spectra of the resuspended colloids confirms the visual impression that the calculated RCF values are indeed 
Table 2 - Calculated RCF values for complete centrifugation, within $30 \mathrm{~min}$., of spherical gold nanoparticles in water, using a liquid height of $3 \mathrm{~cm}$. Experimentally determined nanoparticle recovery in a typical centrifugation/redispersal washing cycle (n.t. $=$ not tested).

\begin{tabular}{lrrl}
\hline diam. & calcd. & exp. & \\
$(\mathrm{nm})$ & RCF & \% recovery & \\
\hline 13 & 11671 & n.t. & \\
20 & 4931 & $94 \quad$ (a) \\
40 & 1233 & n.t. & \\
50 & 789 & 98 & \\
60 & 548 & n.t. \\
80 & 308 & 97 \\
150 & 88 & 97 \\
\hline
\end{tabular}

(a) recovery increases to $97 \%$ by spinning at 1.2 times the calculated RCF

sufficient, and higher speeds for centrifugation are not necessary. In the case of $20 \mathrm{~nm}$ diameter gold spheres, centrifugation at slightly higher acceleration $(\sim 20 \%)$ was required to concentrate all particles near the bottom of the tube (Supporting Information, Figure S7).

Centrifugation at a given RCF corresponds to a sedimentation gradient length scale $z_{0}^{\text {cfg }}$ and corresponding concentration factor $B_{\mathrm{cfg}}$. The gradient length should be small enough, such that the nanoparticles are concentrated well in the pellet. The concentration factor gives an estimate of the concentration increase at the bottom of the tube. For spherical particles, coefficient $s$ and $D$ for Eqn. (15) are obtained from Eqn. (6).

$$
z_{0}^{\text {cfg }}=\frac{D}{s \times g \times \mathrm{RCF}} \quad B_{\mathrm{cfg}}=\frac{z_{\text {tube }}}{z_{0}^{\text {cfg }}\left[1-\exp \left(-z_{\text {tube }} / z_{0}^{\text {cfg }}\right)\right]}
$$

For the conditions in Table $2 z_{0}^{\text {cfg }}$ decreases from $2.1 \mu \mathrm{m}$ (for $13 \mathrm{~nm}$ diameter gold spheres) to well below $1 \mu \mathrm{m}$ (larger particles), which indicates that the particles are well concentrated near the bottom of the tube.

Prolonged centrifugation may concentrate smaller particles of less dense materials. If we take for instance particles of $8 \mathrm{~nm}$ diameter with average density $4.5 \times 10^{3} \mathrm{~kg} \mathrm{~m}^{-3}$ in water (a rough estimate for typical[41] CdSe/ZnS quantum dot particles with a small-molecule capping), then it may be anticipated that these can be concentrated in a liquid pellet less than $300 \mu \mathrm{m}$ high $\left(z_{0}^{\text {cfg }} \sim 31 \mu \mathrm{m}\right)$ by spinning them for 6 hours at $11000 \times g$ (or 12 hours at $5500 \times g$ for a $z_{0}^{\text {cfg }} \sim 63 \mu \mathrm{m}$ ), starting from a $3 \mathrm{~cm}$ liquid height (typical Eppendorf-type vial). Such centrifugation conditions are well in the range of 
standard laboratory centrifuges.

We may use similar reasoning to find the conditions for minimal centrifugal stress, which will correspond to finding the minimum RCF necessary for

a certain pellet compactness ( $\mathrm{small} z_{0}^{\mathrm{cfg}}$ ), and applying prolonged centrifugation, the duration in that case given by $t_{\mathrm{cfg}}$.

Here, we were concerned with centrifugation without density gradient, starting from an initially homogeneous solution. For separation purposes, density gradient methods may be more adapted.[42] Moreover, we used the Mason-Weaver model which assumes a constant gravitational field, instead of the gradient found in centrifugation. Nevertheless, this simple model does provide a means of predicting the behaviour of nanoparticles in solution in a standard laboratory centrifuge, and is therefore relevant for the rational design of nanoparticle purification protocols.

Another implication of this analysis is that the RCFs needed for the centrifugal sedimentation of inorganic nanoparticles and their assemblies is significantly lower than those in analytical ultracentrifuges $(\sim 100000 \times g)$. Analytical centrifugation using lower-speed (and simpler) equipment, such as recent photocentrifuges with space- and time-resolved extinction profiling (STEP), [20-23] is therefore highly relevant for the characterization of inorganic nanoparticle solutions. Compared to the low-cost digital photographybased method presented here, such dedicated and optimised equipment provide more rapid, precise and detailed analysis of nanoparticle samples.

\section{Conclusion}

This work rationalised observations on the settling of nanoparticles in liquid solution in the framework of the Mason-Weaver model. This is illustrated by the quantitative agreement between this model and experimental digital photography for the time-evolution of the concentration gradient of suspended nanoparticles submitted to a gravitational field. A simple experimental protocol for observing the sedimentation process is established, using time-lapse digital photography of the samples in an undisturbed laboratory fridge in order to avoid thermally induced convection.

By fitting a numerical solution of the Mason-Weaver equation to the experimental concentration gradient, the diffusion and sedimentation coefficients $D$ and $s$ are obtained, without need to wait for complete equilibrium to be established. Early studies on sedimentation[3, 43] were mostly concerned with precise measurement of the equilibrium gradient, which only yields the buoyant mass $m_{b}$ of the particle as the sole parameter, not the separate contributions of diffusion and sedimentation coefficients. 
We demonstrated that measuring the evolving concentration gradient of settling solutions distinguishes between individual monomeric nanoparticles and their dimeric assemblies, by giving a distinct combination of diffusion and sedimentation coefficients for each type of nano-object.

In our experiments on gold nanospheres in dilute solution, no significant deviations from the simple Mason-Waver model were observed. Colloidal gold solutions are generally known to be well-behaved in this respect, and we expect many dilute solutions of other non-aggregating (inorganic) nanoparticles to behave in a similar way. Any significant deviations from the predictions made by the Mason-Waver model would point to stronger interparticle interactions, aggregation of individual objects, specific solvation[4-6] or surfactant $[7,8]$ effects, or changes in the properties of the liquid medium. It is important to be aware of such deviations as they may affect other aspects of the behaviour of the nanoparticles in liquid media, such as their interaction with biological entities.

The simple mathematical model and the experimental protocol used here have obvious limitations, and do not substitute advanced analytical centrifugation techniques[14, 16, 19-25, 40]. However, they do provide means for initial and very simple screening of nanoparticle solutions, and rationalise visual observations made at the bench, or upon prolonged storage in the laboratory fridge. The use of a standard digital photo-camera limits the applications to systems that have detectable optical response in the visible. Many particle systems do indeed have such a response, for instance plasmonic materials such as gold, silver, titanium nitride, [44] but also other metal particles, semiconductor quantum dots, [17] and paramagnetic iron oxide.[29] Attainment of equilibrium can take quite some time, and an analytical photocentrifuge[2023] may be a time-saving investment, which also will yield more precise analysis, in particular for broad size distributions and multimodal samples.

Based on the Mason-Weaver model, we obtained simple expressions that give useful recommendations for the centrifugation of nanoparticle solutions, so that delicate solutions can be processed with care, instead of spinning at maximum RCF. The practical insights and simple quantitative expressions provided in the present work are of direct interest for wet-chemical synthesis, purification and application of functional nanoparticle assemblies, and will stimulate further interest in sedimentation analysis[4, 19, 23] for functional nanoparticle solutions. 


\section{Experimental details}

Temperature stabilisation. In a non-thermostated environment such as a lab shelf, even modest temperature changes may create temperature gradients in the sample that lead to convective motion sufficiently strong to prevent the sedimentation equilibrium from establishing itself. Such convective motion plagued early sedimentation experiments, which were concerned with precise measurement of the equilibrium distribution. [3, 43] Thermally driven convection offers an explanation as to why, on a non-thermostated shelf, many colloidal gold solutions seem not to evolve to a sedimentation equilibrium gradient. In the present work, mechanical and thermal perturbations were avoided by carrying out the experiments in a undisturbed laboratory fridge at $277 \mathrm{~K}$.

Quantitative digital photography. In previous work we used quantitative colour imaging for measuring concentration profiles in microfluidic channels, using an optical microscope and a dedicated CCD camera.[45] Here we use a 'consumer-grade' photo camera. Images were taken using a digital camera (Nikon Coolpix P7800, Nikon Corp., Japan) providing the output of unprocessed ('RAW') image data, where the individual pixel values are proportional to the detected light intensity for the particular colour channel. This circumvents problems of linearization generally encountered with most digital cameras.[31] Typically, four samples were photographed in a single picture. A black area was also included to serve as the source for background subtraction.

The 'RAW' image data were read by ImageJ software[46] using the dcraw plugin. [47] The three colour channels of the images were separated (ImageJ) and treated individually as monochrome intensity images. Intensity gradient profiles $I_{\text {raw }}(z)$ for all samples (and all colour channels) in each image of the time series were extracted by horizontal averaging over a rectangular area (typically, the visible optical window of the cells). The pixel values of the black area were averaged for background substraction, $I_{\text {dark }}$. The $z$ scaling was calibrated by precise measurements of specific cell features. Each image frame thus obtained its specific calibration of pixel size. The digital intensity profiles $I_{\text {raw }}(z)$ were then further treated numerically using the Python programming language with scientific extensions.[48-50]

The individual profile traces with their specific $z$ step sizes were resampled at a standard higher resolution in order to have profiles with identical step sizes, facilitating their processing and comparison with theory. Subsequently, background-corrected image profiles $I(z)$ were obtained by subtraction of the near-zero dark background. 


$$
I(z)=I_{\text {raw }}(z)-I_{\text {dark }}
$$

The top area of each extracted $z$ profile, which does not contain liquid, was used for calculating $I_{0}$ by averaging. This corrects slight frame-to-frame variations in illumination intensity. A linear baseline correction, $\mathrm{OD}_{\text {base }}(z)=$ $k_{1} z+k_{2}$, was applied globally to all time-frames for each sample. In all cases, the baseline correction was only modest and not necessary to obtain useful results.

The final corrected optical density is obtained using eqn. (17). We note that we apply this Beer-Lambert-Bouguer formulation despite the condition of monochromatic light not being rigorously fulfilled: the filters used in colour cameras define large spectral bands (width $\sim 100 \mathrm{~nm}$ ). As has been discussed previously, [45] a linear response of the optical density as a function of concentration is still obtained, provided that the extinction spectra of the samples are sufficiently large and their optical density is sufficiently low (under OD $1)$.

$$
\mathrm{OD}(z)=\log _{10}\left(\frac{I_{0}}{I(z)}\right)-\mathrm{OD}_{\text {base }}(z)
$$

Comparison of experiment with theory is achieved by converting the concentration profiles $c(z)$ from the Mason-Weaver model into modeled optical density profiles $\mathrm{OD}_{\text {model }}(z)$ using an "effective extinction coefficient" which we refer here to as $k_{3}$ and can be adapted to rescale the model concentration profile to fit the experimental values.

$$
\mathrm{OD}_{\text {model }}(z)=k_{3} c(z)
$$

Centrifugation. A thermostated bench-top laboratory centrifuge (Hettich Mikro 220r with 1195-A rotor) was used. The relation between rotational speed (rounds per minute, RPM) and relative centrifugal force (RCF) is

$$
\mathrm{RCF}=1.12 \times 10^{-3} \times R \times(\mathrm{RPM})^{2}
$$

The rotor radius $R$ for the $1195-\mathrm{A}$ rotor used is $0.087 \mathrm{~m}$. The maximum speed for this rotor is $18000 \mathrm{RPM}$, corresponding to an RCF of $31500 \times \mathrm{g}$. Samples were generally centrifuged with the centrifuge thermostat at $298 \mathrm{~K}$.

Colloidal gold solutions. For the study of the sedimentation of gold nanospheres (Section 3) we used standard aqueous solutions of colloidal gold from commercial sources (BBI Solutions, UK, and Sigma-Aldrich, France) and samples synthesised and characterised according to literature procedures. [51] 
All colloids are stabilised with negatively charged carboxylate ligands, and the particles have negative zeta potential. The samples were diluted with pure water where necessary. Typical optical densities at the extinction maximum were in the range $0.3 \ldots 1$.

The carboxylate ligand layers around the particles are thin compared to the particle diameter $(d>20 \mathrm{~nm})$, and do not contribute significantly to the buoyant mass. For smaller particles $(<10 \mathrm{~nm})$ and larger ligands such effects may become significant, and will then show up as a deviation from the idealised predicted behaviour, and result in a change in effective buoyant mass, and effective diffusion and sedimentation coefficents. Furthermore, solvation effects on sedimentation may become significant, as the shell of solvent molecules around the object influences its hydrodynamic behaviour. [4-6]

DNA-linked dimers. The dimers were constructed from $13 \mathrm{~nm}$ gold nano-

Gold nanoparticles of diameter $13 \mathrm{~nm}$ were synthesised according to the published protocol. [52, 53] Briefly, a solution of sodium tetrachloroaurate (50 $\mathrm{mL}, 1 \mathrm{mM}$ ) was heated up to $100{ }^{\circ} \mathrm{C}$ under stirring. Once boiling, a solution of sodium citrate $(5 \mathrm{~mL}, 2 \mathrm{wt} . \%)$ was added. After appearance of the typical red colour, boiling and stirring were maintained for 15 additional minutes before letting cool down to room temperature. To stabilise the AuNPs, BSPP was added $(15 \mathrm{mg})$ and the solution was left to stir overnight. TEM images were obtained with a Hitachi H7000 transmission electron microscope, operating at $75 \mathrm{kV}$ bias. All samples preparation involved deposition and evaporation of a specimen droplet on a carbon film-coated 400 Mesh copper grid. TEM analysis is shown in the Supporting Information, Fig. S2.

Dimers of $13 \mathrm{~nm}$ AuNPs were synthesised using DNA hybridisation. [54] BSPP-coated AuNPs of $13 \mathrm{~nm}$ (5 pmol) were flocculated using $\mathrm{NaCl}$ and centrifuged for $5 \mathrm{~min}$ at RCF $25000 \times g$ (Eppendorf centrifuge 5417R, rotor FA-45-24-11, $8.8 \mathrm{~cm}$ radius). The supernatant was taken off and the particles were re-dispersed in $100 \mu \mathrm{L}$ buffer $(20 \mathrm{mM}$ phosphate, $5 \mathrm{mM} \mathrm{NaCl}$ ). DNA single strands $\mathbf{S} 1$ respectively $\mathbf{S} 2$ (15 pmol) were added each to a separate nanoparticle solution to achieve a ratio DNA/particles of 3:1. A solution of BSSP $(10 \mu \mathrm{L}, 1 \mathrm{mg} / 20 \mu \mathrm{L})$ was added and the reaction mixture was shaken for $1 \mathrm{~h}$, in order to deprotect the thiol group on the DNA and to allow its attachment to the particles. The AuNPs were then centrifuged for $15 \mathrm{~min}$ at $25000 \times g$ and redispersed in hybridization buffer $(50 \mu \mathrm{L}, 6 \mathrm{mM}$ phosphate, $80 \mathrm{mM} \mathrm{NaCl}$ ). After mixing the two types of particles (S1 and $\mathbf{S} 2$ strands), a complementary DNA strand $\mathbf{S} 3(2.5 \mathrm{pmol})$ was added to create the dimers. Hybridisation was realized by heating up the solution to $70^{\circ} \mathrm{C}$ and leaving it to cool down slowly and gradually to room temperature. The dimers were purified by agarose gel electrophoresis (1.75\% agarose gel in $0.5 \mathrm{x}$ TBE, 1 
$\mathrm{h}$ at $90 \mathrm{~V})$. After extraction from the gel the solution was centrifuged for 10 min at $25000 \times g$ and the dimers were redisolved in Milli-Q water.

\section{Acknowledgements}

This work was supported by Dstl (UK) in the framework of the France-UK Ph.D. programme. MW acknowledges funding by the Agence Nationale de la Recherche (France), grant ANR-2010-JCJC-1005-1 (COMONSENS).

\section{References}

[1] M. D. Haw. J. Phys.: Condens. Matter 2002, 14, 337769.

[2] P. Ball. Chemistry World 2005, 238.

[3] J. Perrin. J. Phys. Theor. Appl. 1910, 95.

[4] J. W. Williams, K. E. Van Holde, R. L. Baldwin, H. Fujita. Chem. Rev. 1958, 58, 4715 .

[5] T. M. Laue, W. F. Stafford III. Annu. Rev. Biophys. Biomol. Struct. 1999, 28, 175 .

[6] J.-J. Huang, Y. J. Yuan. Phys. Chem. Chem. Phys. 2016, 18, 1712312.

[7] J. T. Li, K. D. Caldwell. Langmuir 1991, 7, 102034.

[8] M. Andersson, K. Fromell, E. Gullberg, P. Artursson, K. D. Caldwell. Anal. Chem. 2005, 77, 175488.

[9] V. Sharma, K. Park, M. Srinivasarao. Proc. Nat. Acad. Sci. 2009, 106, 134981.

[10] K. Park, H. Koerner, R. A. Vaia. Nano Lett. 2010, 10, 41433.

[11] L. Scarabelli, M. Coronado-Puchau, J. J. Giner-Casares, J. Langer, L. M. Liz-Marzán. ACS Nano 2014, 8, 65833.

[12] F. Liebig, R. M. Sarhan, C. Prietzel, A. Reinecke, J. Koetz. RSC Adv. 2016, 6, 4033561 .

[13] E. C. Cho, Q. Zhang, Y. Xia. Nat. Nanotechnol. 2011, 6, 6385. 
[14] J. M. Zook, V. Rastogi, R. I. MacCuspie, A. M. Keene, J. Fagan. ACS Nano 2011, 5, 108070.

[15] R. P. Carney, J. Y. Kim, H. Qian, R. Jin, H. Mehenni, F. Stellacci, O. M. Bakr. Nat. Commun. 2011, 2, May 335.

[16] K. L. Planken, H. Cölfen. Nanoscale 2010, 2, 101849.

[17] B. Demeler, T.-L. Nguyen, G. E. Gorbet, V. Schirf, E. H. Brookes, P. Mulvaney, A. O. El-Ballouli, J. Pan, O. M. Bakr, A. K. Demeler, B. I. Hernandez Uribe, N. Bhattarai, R. L. Whetten. Anal. Chem. 2014, 86, 157688 .

[18] J. Walter, K. Löhr, E. Karabudak, W. Reis, J. Mikhael, W. Peukert, W. Wohlleben, H. Cölfen. ACS Nano 2014, 8, 98871.

[19] M. J. Urban, I. T. Holder, M. Schmid, V. Fernandez Espin, J. Garcia de la Torre, J. S. Hartig, H. Cölfen. ACS Nano 2016, 107418.

[20] T. Detloff, T. Sobisch, D. Lerche. Part. Part. Syst. Charact. 2006, 23 184.

[21] T. Detloff, T. Sobisch, D. Lerche. Powder Technol. 2007, 17450.

[22] E. Ibrahim, S. Hampel, J. Thomas, D. Haase, A. U. B. Wolter, V. O. Khavrus, C. Täschner, A. Leonhardt, B. Büchner. J. Nanoparticle Res. 2012, 141118.

[23] J. Walter, T. Thajudeen, S. Sü $\beta$, D. Segets, W. Peukert. Nanoscale 2015, 76574 .

[24] Ž. Krpetić, A. M. Davidson, M. Volk, R. Lévy, M. Brust, D. L. Cooper. ACS Nano 2013, 7, 108881.

[25] A. Knauer, A. Thete, S. Li, H. Romanus, A. Csáki, W. Fritzsche, J. M. Köhler. Chem. Eng. J. 2011, 166, 31164.

[26] C. M. Alexander, J. C. Dabrowiak, J. Goodisman. J. Coll. Interf. Sci. 2013, 39653.

[27] C. M. Alexander, J. Goodisman. J. Coll. Interf. Sci. 2014, 418103.

[28] A. I. López-Lorente, M. Sieger, M. Valcárcel, B. Mizaikof. Anal. Chem. 2014, 86783 . 
[29] V. Prigiobbe, S. Ko, C. Huh, S. L. Bryant. J. Coll. Interf. Sci. 2015, 44758.

[30] M. Stevens, C. A. Páraga, I. C. Cuthill, J. C. Partridge, T. S. Troscianko. Biol. J. Linn. Soc. 2007, 90, 211.

[31] J. E. Garcia, A. G. Dyer, A. D. Greentree, G. Spring, P. A. Wilksch. PLoS ONE 2013, 8, 11 e79534.

[32] T. Schwaebel, O. Trapp, U. H. F. Bunz. Chem. Sci. 2013, 4, 1273.

[33] M. Mason, W. Weaver. Phys. Rev. 1924, 23412.

[34] O. Lamm. Ark. Mat. Astron. Fys. 1929, $21 B 1$.

[35] P. H. Brown, P. Schuck. Comp. Phys. Commun. 2008, 178, 2105.

[36] P. Schuck. Biophys. J. 2000, 78, 31606.

[37] W. Weaver. Phys. Rev. 1926, 27, 4499.

[38] K. E. Van Holde, R. L. Baldwin. J. Phys. Chem. 1958, 62, 6734.

[39] J. R. G. Navarro, M. H. V. Werts. Analyst 2013, 138583.

[40] J. B. Falabella, T. J. Cho, D. C. Ripple, V. A. Hackley, M. J. Tarlov. Langmuir 2010, 26, 1512740.

[41] A. R. Clapp, E. R. Goldman, H. Mattoussi. Nat. Protoc. 2006, 1, 3 1258 .

[42] B. Kowalczyk, I. Lagzi, B. A. Grzybowski. Curr. Opinion Coll. Interf. Sci. 2011, 16, 2135.

[43] N. Johnston, L. G. Howell. Phys. Rev. 1930, 35274.

[44] U. Guler, S. Suslov, A. V. Kildishev, A. Boltasseva, V. M. Shalaev. Nanophotonics 2015, 4, 3269.

[45] M. H. V. Werts, V. Raimbault, R. Texier-Picard, R. Poizat, O. Français, L. Griscom, J. R. G. Navarro. Lab. Chip 2012, 12808.

[46] C. A. Schneider, W. S. Rasband, K. W. Eliceiri. Nat. Methods 2012, 9 671.

[47] D. Coffin. dcraw. URL http://www. cybercom.net/ coffin/dcraw/. 
[48] K. J. Millman, M. Aivazis. Comp. Sci. Eng. 2011, 13, 29.

[49] S. Van Der Walt, S. C. Colbert, G. Varoquaux. Comp. Sci. Eng. 2011, 13, 222 .

[50] J. D. Hunter. Comp. Sci. Eng. 2007, 990.

[51] N. G. Bastús, J. Comenge, V. Puntes. Langmuir 2011, 27, 1711098.

[52] J. Turkevich, P. C. Stevenson, J. Hillier. Discuss. Faraday Soc. 1951, 11, c 55 .

[53] J. Turkevich, P. C. Stevenson, J. Hillier. J. Phys. Chem. 1953, 57, 7 670.

[54] A. Heuer-Jungemann, R. Kirkwood, A. H. El-Sagheer, T. Brown, A. G. Kanaras. Nanoscale 2013, 5, 167209. 


\section{Supporting Information}

for Part. Part. Syst. Charact. 2017, 1700095

DOI: $10.1002 /$ ppsc201700095

The sedimentation of colloidal nanoparticles in solution and its study using quantitative digital photography

Johanna Midelet, Afaf H. El-Sagheer, Tom Brown, Antonios G. Kanaras and Martinus H. V. Werts*

\section{S.1 Synthesis of DNA-linked gold nanosphere dimers}

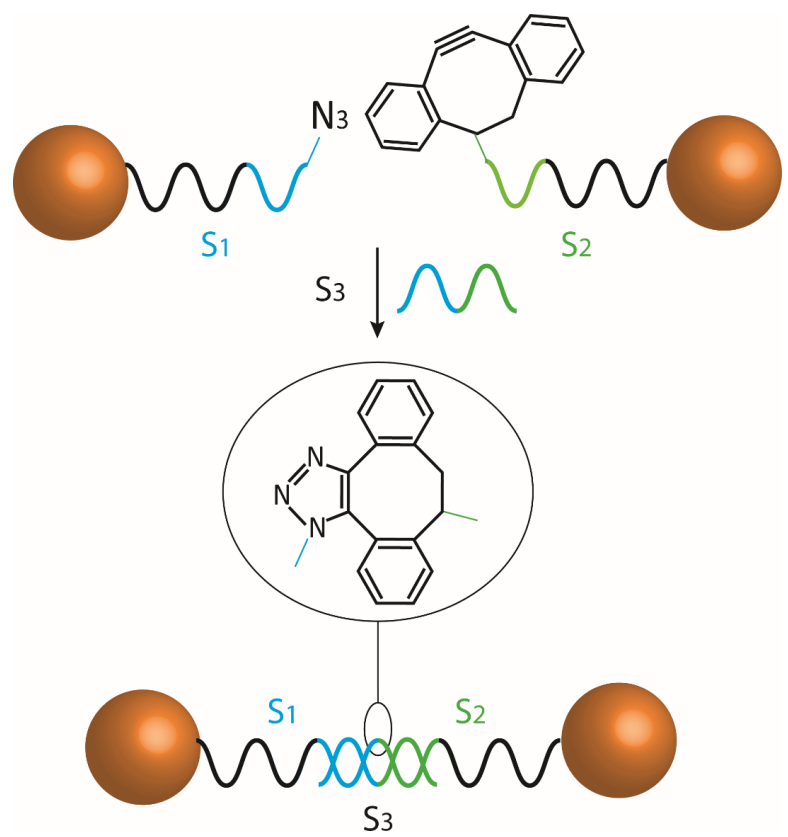

Figure S1 - Schematic of the assembly of DNA-linked dimers and subsequent 'click' chemistry. 
Table S1 - Sequences of single DNA strands S1, S2 and S3.

\begin{tabular}{|c|c|}
\hline Abbreviation & DNA sequence and modifications \\
\hline $\mathrm{S}_{1}$ (azide derivative $\mathrm{X} 1$ ) & $\mathbf{5}^{\prime}-\left(\mathrm{C}_{6} \mathrm{H}_{12} \mathrm{~S}\right)$ AAAAAAAAACGAGTGCTAAGGATCCGAAX1 \\
$\mathrm{S}_{2}$ (alkyne derivative $\mathrm{X}$ 2) & $\mathbf{3}^{\prime}-\left(\mathrm{C}_{6} \mathrm{H}_{12} \mathrm{~S}\right)$ AAAAAAAAGCTTACCTATAGACGTCACTTX2 \\
$\mathrm{S}_{3}$ (Splint) & $\mathbf{5}^{\prime}-$ ACACACCGAATGGATATCTGCAGTGAATTCGGATCCTTAGCACTCGACACAC \\
\hline
\end{tabular}
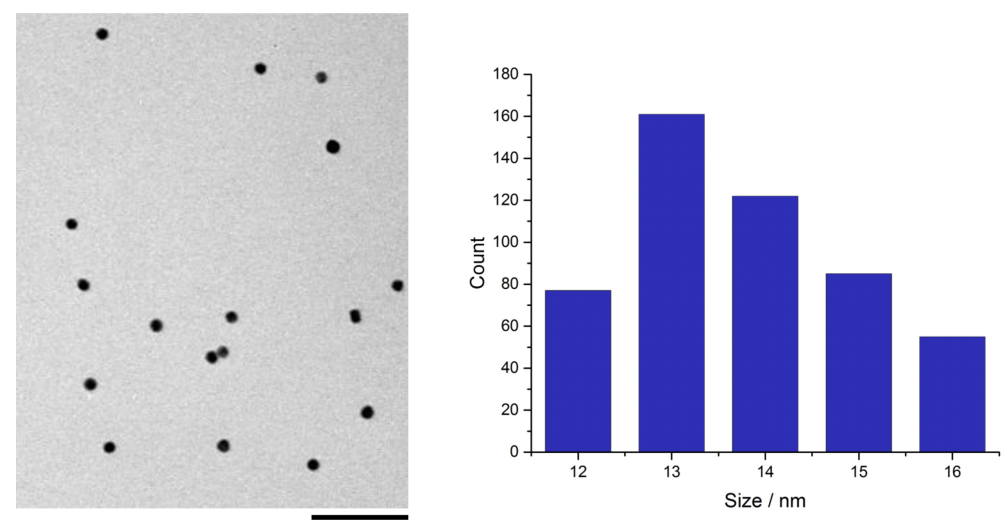

Figure S2 - Citrate-stabilised gold nanoparticles used for the construction of DNA-linked dimers. a) TEM image of $13 \pm 2 \mathrm{~nm}$ spherical gold nanoparticles. Scale bar is $100 \mathrm{~nm}$. b) Corresponding size distribution histogram, $N=500$ particles 


\section{S.2 Finite-difference solver for the Mason-Weaver equa- tion}

\section{S.2.1 Dimensionless Mason-Weaver equation}

A numerical solver has been designed for the dimensionless version of the Mason-Weaver equation. Conversion between the Mason-Weaver equation and its dimensionless form can be achieved using:

$$
\zeta=z / z_{0} \quad z_{0}=\frac{D}{s g}
$$

and

$$
\tau=t / t_{0} \quad t_{0}=\frac{D}{s^{2} g^{2}}
$$

The aim is now to obtain the time-evolving spatial concentration distribution $c(\zeta, \tau)$ obeying the dimensionless Mason-Weaver equation (S3), starting from an arbitrary initial concentration profile $c(\zeta, 0)$.

$$
\begin{gathered}
\frac{\partial c}{\partial \tau}=\frac{\partial^{2} c}{\partial \zeta^{2}}+\frac{\partial c}{\partial \zeta} \\
\frac{\partial c}{\partial \zeta}+c=0 \quad @ \zeta=0, \zeta=\zeta_{\max }
\end{gathered}
$$

A finite-difference scheme for numerically solving this equation has been proposed previously, [26] but unfortunately, in our hands, this did not yield a working computer code. In particular, the proposed time coordinate transformation leads to unsurmountable numerical problems in our implementation. Here, we propose a different finite-difference approximation that yields a stable, robust numerical solver, that moreover does not suffer from mass conservation problems reported[26] for the previously proposed scheme.

\section{S.2.2 Crank-Nicolson scheme}

Time and space are discretised into $N+1$ and $J+1$ grid points, respectively. Space is on an evenly spaced grid between 0 and $\zeta_{\max }$.

$$
\begin{gathered}
\zeta_{j}=j \Delta \zeta \quad(j=0,1 \ldots J) \\
\Delta \zeta=\frac{\zeta_{\max }}{J}
\end{gathered}
$$


Time may be on an evenly or unevenly spaced grid. We use an exponentially expanding grid, going from 0 to $\tau_{\max }$.

$$
\begin{gathered}
\tau_{n}=\exp (k n)-1 \quad(n=0,1 \ldots N) \\
k=\frac{\ln \left(\tau_{\max }+1\right)}{N}
\end{gathered}
$$

We make the following Crank-Nicolson style finite-difference approximations for the PDE, where $c_{j}^{n}$ is the value of $c\left(\zeta_{j}, \tau_{n}\right)$.

$$
\begin{gathered}
\frac{\partial c}{\partial \tau} \rightarrow \gamma\left(c_{j}^{n+1}-c_{j}^{n}\right) \\
\frac{\partial^{2} c}{\partial \zeta^{2}} \rightarrow \alpha\left(c_{j+1}^{n}-2 c_{j}^{n}+c_{j-1}^{n}+c_{j+1}^{n+1}-2 c_{j}^{n+1}+c_{j-1}^{n+1}\right) \\
\frac{\partial c}{\partial \zeta} \rightarrow \beta\left(c_{j+1}^{n}-c_{j-1}^{n}+c_{j+1}^{n+1}-c_{j-1}^{n+1}\right)
\end{gathered}
$$

where (noting the factors 2 and 4 in $\alpha$ and $\beta$ )

$$
\gamma=\frac{1}{\tau_{n+1}-\tau_{n}} \quad \alpha=\frac{1}{2(\Delta \zeta)^{2}} \quad \beta=\frac{1}{4 \Delta \zeta}
$$

With these finite-difference approximations, and after rearrangement of the terms, the discrete Mason-Weaver equation can be written as follows $(j=1,2 \ldots J-1)$.

$$
\begin{aligned}
& (-\alpha+\beta) c_{j-1}^{n+1}+(\gamma+2 \alpha) c_{j}^{n+1}+(-\alpha-\beta) c_{j+1}^{n+1} \\
& \quad=(\alpha-\beta) c_{j-1}^{n}+(\gamma-2 \alpha) c_{j}^{n}+(\alpha+\beta) c_{j+1}^{n}
\end{aligned}
$$

The boundary conditions are set as follows. At $\zeta=0$ we choose

$$
\begin{gathered}
c \rightarrow \frac{1}{4}\left(c_{0}^{n}+c_{0}^{n+1}+c_{1}^{n}+c_{1}^{n+1}\right) \\
\frac{\partial c}{\partial \zeta} \rightarrow 2 \beta\left(c_{1}^{n}-c_{0}^{n}+c_{1}^{n+1}-c_{0}^{n+1}\right)
\end{gathered}
$$

Approximation of $c$ using (S13) (instead of simply taking the values at $c_{0}$ ) leads to much better behaviour in terms of mass conservation (the sum over all $c_{j}$ ), in particular at longer times.

After substitution and rearrangement this results in 


$$
\left(-2 \beta+\frac{1}{4}\right) c_{0}^{n+1}+\left(2 \beta+\frac{1}{4}\right) c_{1}^{n+1}=\left(2 \beta-\frac{1}{4}\right) c_{0}^{n}+\left(-2 \beta-\frac{1}{4}\right) c_{1}^{n}
$$

At $\zeta=\zeta_{\max }$ the equivalent choice was made (using backward difference instead of forward difference, naturally).

The equations (discretised PDE and BCs) are assembled into a matrix equation involving tridiagonal matrices. Numerically solving this equation yields the $c_{j}^{n+1}$ from $c_{j}^{n}$, i.e. the concentration profile at time $\tau_{n+1}$ using the concentration profile from time $\tau_{n}$. Repeating the process, starting from the initial condition at $\tau_{0}$, yields the evolution of the concentration profile, obeying the Mason-Weaver equation.

\section{S.2.3 Implementation}

The finite-difference scheme was implemented using Python (with numpy for array manipulation and sparse matrix routines from scipy). For brevity, we only give the solver code without any plotting, processing or storage of results. It consists of a single loop that generates the successive concentration profiles $c_{j}^{n}$ at each $\tau_{n}$, starting from the initial conditions at $\tau_{0}$.

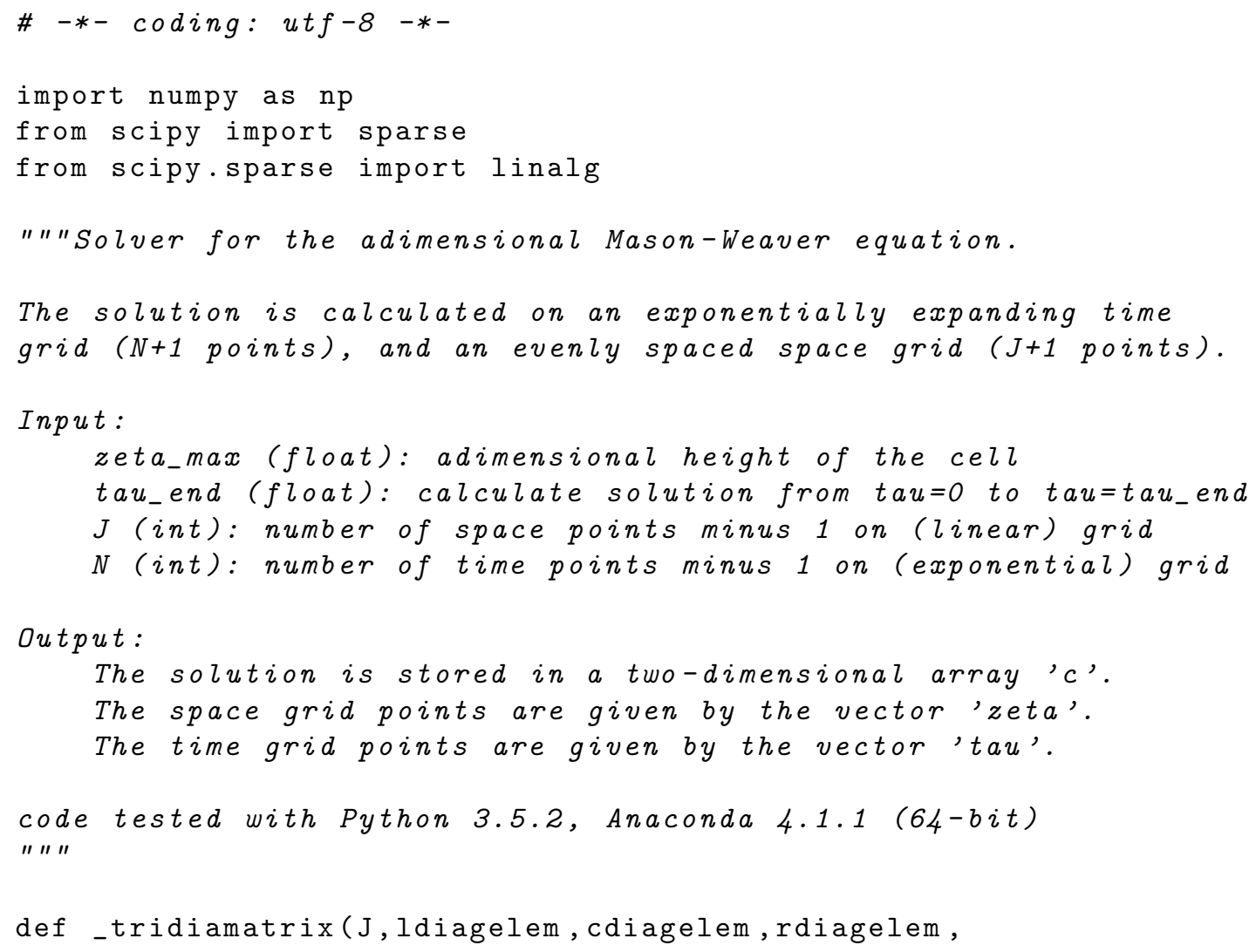




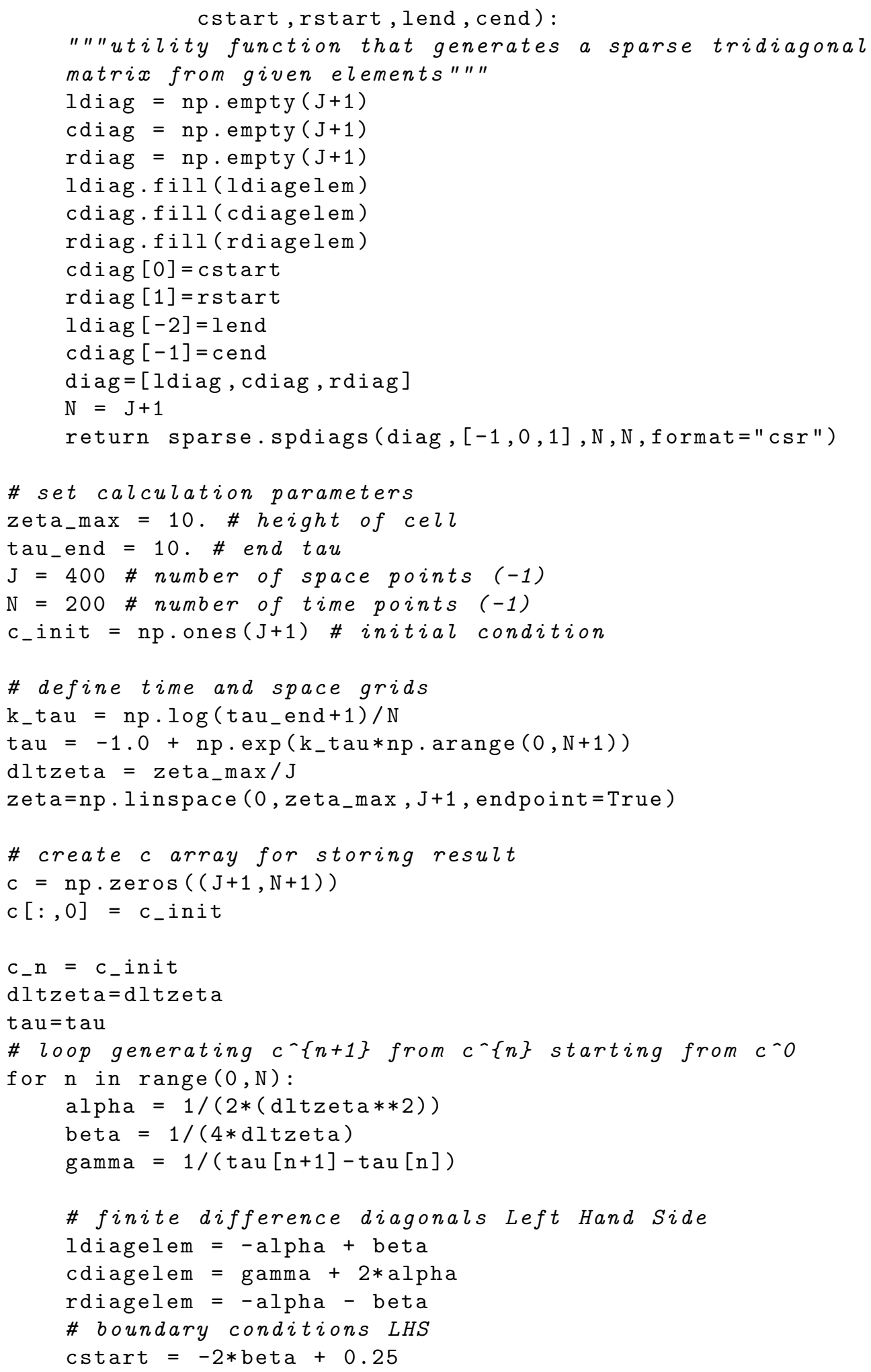




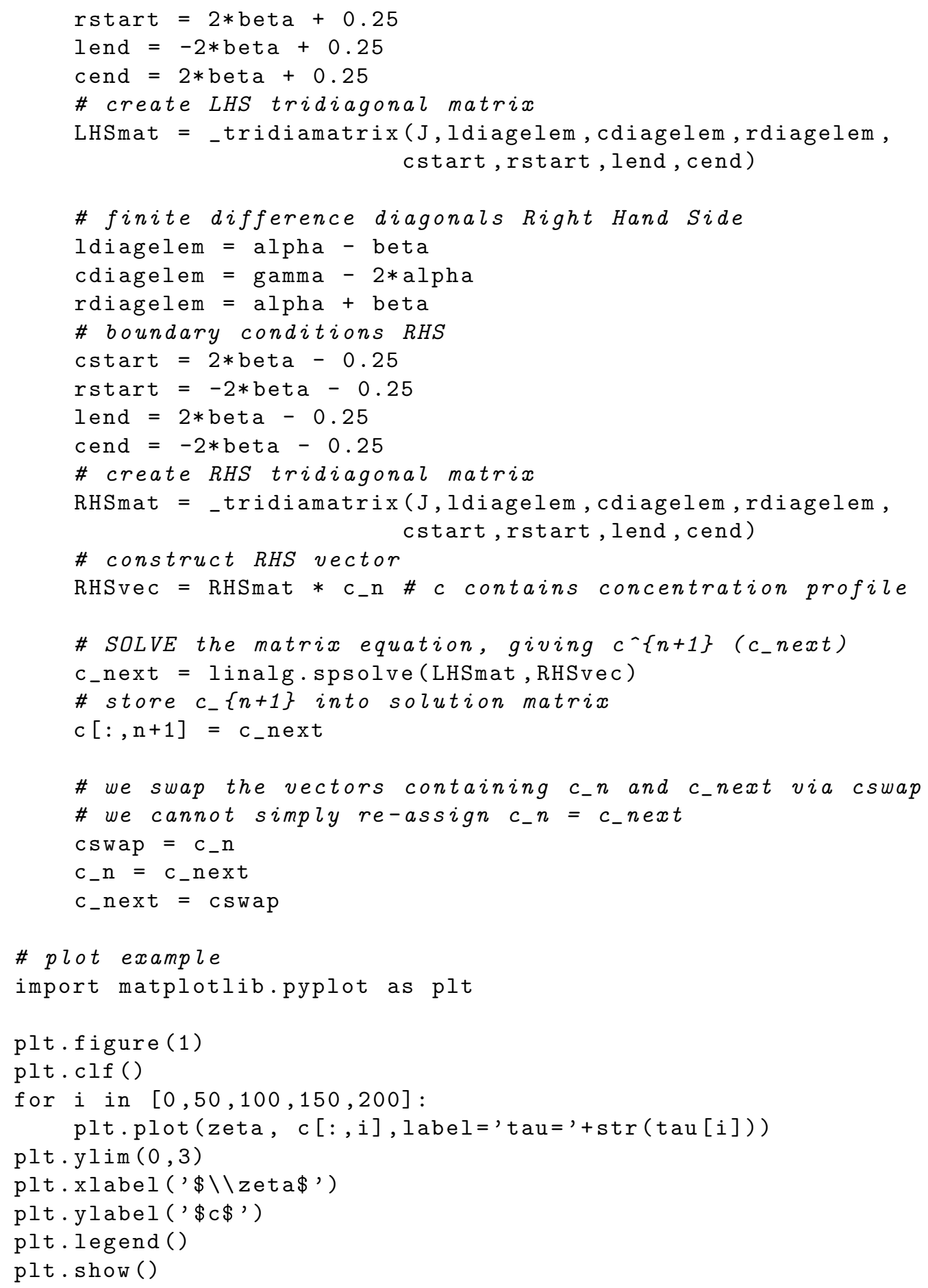




\section{S.2.4 Validation of the numerical solution}

The numerical solver was tested extensively for a wide range of $\zeta_{\max }$ and $\tau$. Typical values for $N$ and $J$ were on the order of several hundred grid points. Numerical mass conservation and convergence of the gradient to the analytical Boltzmann equilibrium solution were verified.

An example of a numerical solution is given in Figure S3, which also illustrates that the numerical solution faithfully converges to the analytical equilibrium distribution, for $\tau=1.4 \tau_{\text {sed }}$.
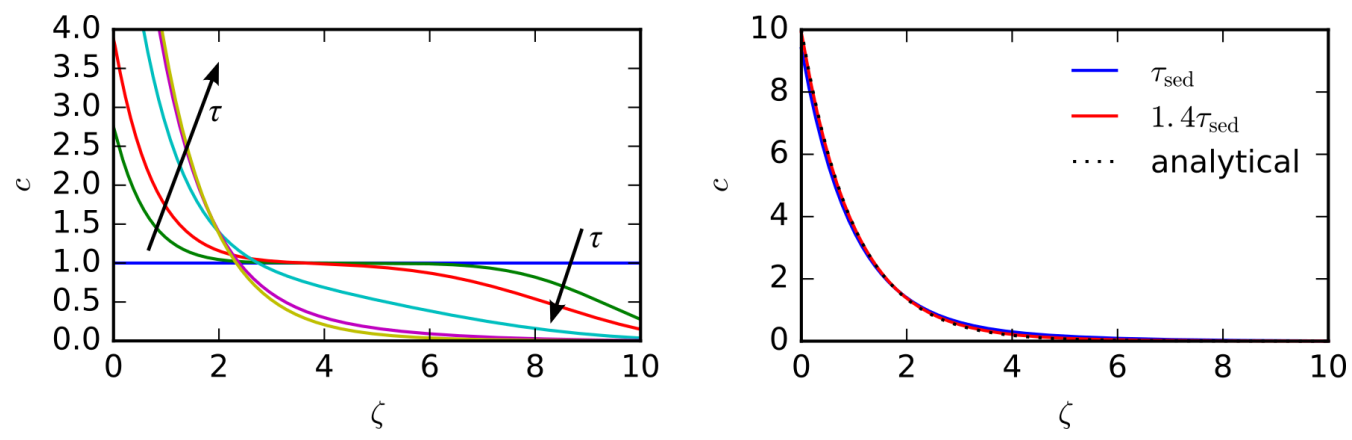

Figure S3 - Left: Numerical solution $(N=200, J=400)$ to the dimensionless Mason-Weaver equation with $\zeta_{\max }=10$ and $\tau$ going from 0 to 30. Right: Comparison of the analytical equilibrium profile and the numerical solution at longer times, demonstrating (1) that the numerical solution converges to the analytical equilibrium (Boltzmann) distribution and (2) that at $1.4 \tau_{\text {sed }}$ the system is virtually at equilibrium.

Mass conservation is observed in the numerical scheme, as illustrated in Figure S4. A slight increase of the total numerical mass is observed, but the numerical solution does not diverge at longer simulation times, as a result of our choice for the boundary conditions, cf. Equation (S13). 


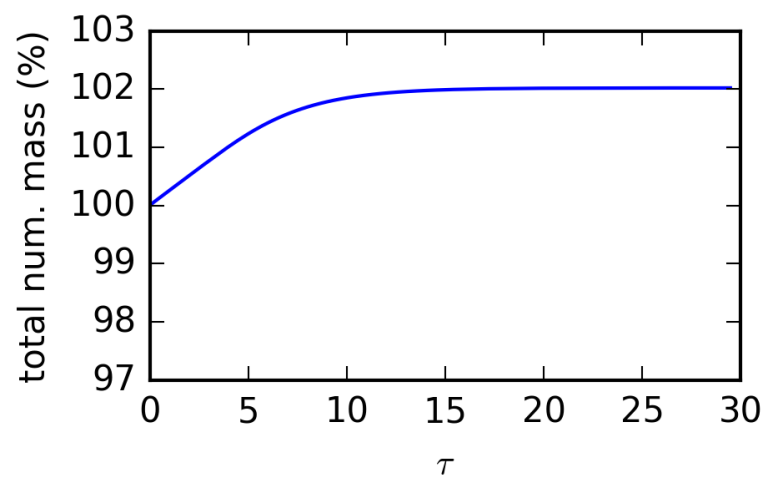

Figure S4 - Evolution of the total mass in the numerically simulated system $\left(N=200, J=400, \zeta_{\max }=10, \tau=0 \ldots 30\right)$, obtained by summing all space grid points. A slight increase $(<2 \%)$ of the total numerical mass is observed, but the total mass does not diverge for long simulation times. 


\section{S.3 Sedimentation parameters for gold nanospheres in water}

Table S2 - Calculated diffusion $D$ and sedimentation $s$ coefficients for gold nanoparticles in water at two different temperatures.

\begin{tabular}{lcccc}
\hline & \multicolumn{2}{c}{$298 \mathrm{~K}$} & \multicolumn{2}{c}{$277 \mathrm{~K}$} \\
& \multicolumn{2}{c}{$0.89 \mathrm{mPa} . \mathrm{s}$} & \multicolumn{2}{c}{$1.56 \mathrm{mPa} . \mathrm{s}$} \\
\cline { 2 - 5 } diam. & \multicolumn{1}{c}{$D$} & $\mathrm{~s}$ & $\mathrm{D}$ & $s$ \\
$(\mathrm{~nm})$ & $10^{-12} \mathrm{~m}^{2} \mathrm{~s}^{-1}$ & $10^{-9} \mathrm{~s}$ & $10^{-12} \mathrm{~m}^{2} \mathrm{~s}^{-1}$ & $10^{-9} \mathrm{~s}$ \\
\hline 13 & 37.8 & 0.193 & 19.9 & 0.110 \\
20 & 24.5 & 0.457 & 13.0 & 0.260 \\
40 & 12.3 & 1.83 & 6.48 & 1.04 \\
50 & 9.82 & 2.86 & 5.18 & 1.62 \\
60 & 8.18 & 4.12 & 4.32 & 2.34 \\
80 & 6.14 & 7.32 & 3.24 & 4.16 \\
100 & 4.91 & 11.4 & 2.59 & 6.50 \\
150 & 3.27 & 25.7 & 1.73 & 14.6 \\
\hline
\end{tabular}

Table S3 - Values of equilibrium gradient length scale $z_{0}$ (in $\mathrm{mm}$ ) for spherical gold nanoparticles in water, at two different temperatures (room temperature and fridge)

\begin{tabular}{lrr}
\hline diam. & \multicolumn{2}{c}{$z_{0}(\mathrm{~mm})$} \\
$(\mathrm{nm})$ & $298 \mathrm{~K}$ & $277 \mathrm{~K}$ \\
\hline 13 & 19.9 & 18.5 \\
20 & 5.147 & 5.08 \\
40 & 0.684 & 0.636 \\
50 & 0.350 & 0.325 \\
60 & 0.203 & 0.188 \\
80 & 0.0855 & 0.0794 \\
100 & 0.0438 & 0.0407 \\
150 & 0.0130 & 0.0121 \\
\hline
\end{tabular}


Table S4 - Calculated concentration factor $B$ for spherical gold nanoparticles in water at $277 \mathrm{~K}$ (left columns) and $298 \mathrm{~K}$ (right columns), for cell heights $\left(z_{\max }\right)$ of $1 \mathrm{~mm}$ and $1 \mathrm{~cm}$

\begin{tabular}{lcccc}
\hline & \multicolumn{2}{c}{$277 \mathrm{~K}$} & \multicolumn{2}{c}{$298 \mathrm{~K}$} \\
\cline { 2 - 5 } diam. & \multicolumn{3}{c}{$B$} \\
$(\mathrm{~nm})$ & $1 \mathrm{~mm}$ & $1 \mathrm{~cm}$ & $1 \mathrm{~mm}$ & $1 \mathrm{~cm}$ \\
\hline 13 & 1.03 & 1.29 & 1.03 & 1.27 \\
20 & 1.10 & 2.29 & 1.09 & 2.18 \\
40 & 1.99 & 15.7 & 1.90 & 14.6 \\
50 & 3.22 & 30.7 & 3.03 & 28.6 \\
60 & 5.34 & 53.1 & 4.97 & 49.4 \\
80 & 12.6 & 126 & 11.7 & 117 \\
100 & 24.6 & 246 & 22.9 & 229 \\
150 & 83.0 & 830 & 77.1 & 771 \\
\hline
\end{tabular}

Table S5 - Calculated sedimentation times (in hours) for gold nanoparticles in water for a cell with $z_{\max }=1 \mathrm{~cm}$. A good rough estimate for the time to reach equilibrium is $t_{\text {equil }} \approx 1.4 t_{\text {sed }}$. These times are directly proportional to cell height.

\begin{tabular}{lcc}
\hline diam. & \multicolumn{2}{c}{$t_{\text {sed }}(h)$} \\
$(\mathrm{nm})$ & $298 \mathrm{~K}$ & $277 \mathrm{~K}$ \\
\hline 13 & 1465 & 2580 \\
20 & 619 & 1090 \\
40 & 155 & 272 \\
50 & 99.0 & 174 \\
60 & 68.8 & 121 \\
80 & 38.7 & 68.1 \\
100 & 24.8 & 43.6 \\
150 & 11.0 & 19.4 \\
\hline
\end{tabular}




\section{S.4 The time needed to reach sedimentation equilib- rium}

In the main text we state that a simple but robust approximation for the time to reach sedimentation equilibrium is 1.4 times the time $t_{\text {sed }}$ needed by a particle to traverse the liquid column from top to bottom at its terminal sedimentation velocity. This approximation enables a simple estimate of the time needed for centrifugation of nanoparticles at a certain centrifugal acceleration.

A more sophisticated approach to this question is known in the ultracentrifugation literature.[38] This approach by Van Holde and Baldwin[38] yields the time to arrive near equilibrium within a 'distance' determined by parameter $\epsilon$. The choice for this parameter made in [38] is based on the difference in particle concentration between the top and the bottom of the cell, $\Delta c=c(0)-c\left(z_{\max }\right)$.

$$
\epsilon=1-\frac{\Delta c(t)}{\Delta c_{\mathrm{eq}}}
$$

At the beginning, when the concentration is homogenous, $\epsilon=1$. As sedimentation equilibrium is reached $\epsilon$ will tend to zero. A close approach to equilibrium is typically obtained for $\epsilon<0.02$. The time to come within this 'distance' $\epsilon$ from equilibrium can then be approximated with the following expression.

$$
\begin{gathered}
t_{\text {equil }} \approx \frac{z_{\text {max }}^{2}}{D} F(\alpha) \\
F(\alpha)=-\frac{1}{\pi^{2} U(\alpha)} \ln \left(\frac{\pi^{2} U^{2}(\alpha) \epsilon}{4[1+\cosh (1 / 2 \alpha)]}\right) \\
U(\alpha)=1+\frac{1}{4 \pi^{2} \alpha^{2}}
\end{gathered}
$$

The time to reach equilibrium is expressed as a function of $\alpha$, which, in the context of the present work, is given by

$$
\alpha=\frac{k_{\mathrm{B}} T}{m_{b} g z_{\max }}
$$

An interesting point is that the parameter $\alpha$ can also be expressed as the ratio from the length scale of the equilibrium gradient $z_{0}$ to the height of the liquid column $z_{\max }$. 


$$
\alpha=\frac{k_{\mathrm{B}} T}{m_{b} g z_{\max }}=\frac{z_{0}}{z_{\max }}
$$

It is thus a measure of how the equilibrium gradient 'fits' in the height of the liquid column. For 'oversized' containers (where all particles will finally be concentrated near the bottom of the container), the attainment of equilibrium for initial homogeneous distribution will be governed by the time needed for sedimentation, whereas containers much smaller than the equilibrium gradient will not show much of the gradient, i.e. when $\alpha$ becomes very large, the gradient will be undetectable.

Equation (S17) may be expressed as the ratio of $t_{\text {equil }}$ and $t_{\text {sed }}$. It can be shown that this ratio is a pure function of $\alpha$. It thus depends only on the ratio $z_{0} / z_{\max }$, not on $D$ and $s$ individually.

$$
\frac{t_{\text {equil }}}{t_{\text {sed }}}=\frac{F(\alpha)}{\alpha}
$$

It is important to note that Equation (S17) is an approximation for $\alpha>0.1,[38]$ and that it is not valid at smaller values. For small $\alpha$ our approximation $t_{\text {equil }} \approx 1.4 t_{\text {sed }}$ is valid and safe. For $\alpha>0.1$, we find that the time predicted by Eqn (S17) is always shorter than the rough $1.4 t_{\text {sed }}$ estimation (see Figure S5)
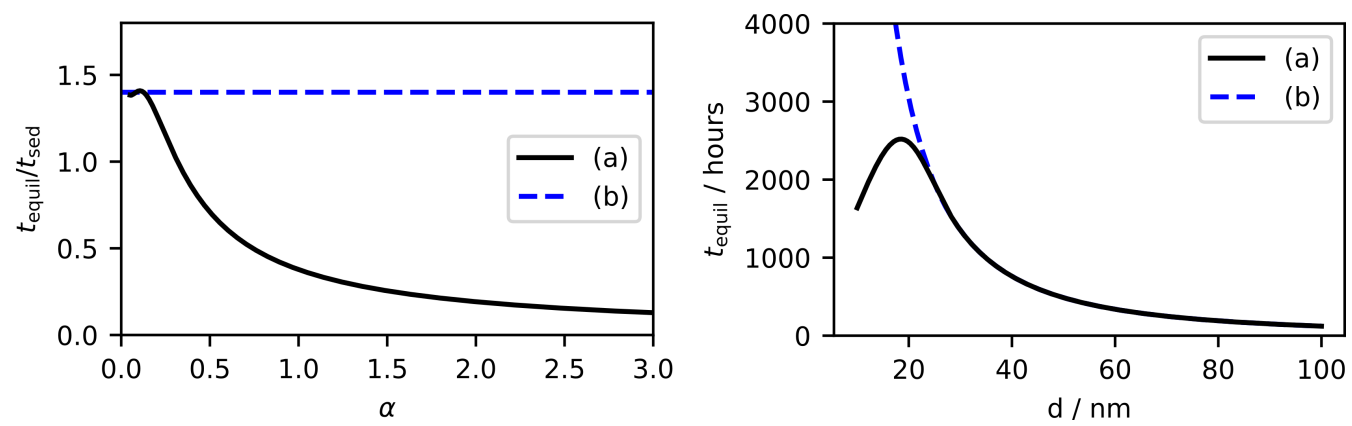

Figure S5 - Left: The ratio of the 'time-to-equilibrium' $t_{\text {equil }}$ and the 'sedimentation time' $t_{\text {sed }}$ as a function of parameter $\alpha$. Curves (a) are calculated using Eqn. (S17), valid for $\alpha>0.1$. We chose $\epsilon=0.018$, such that the maximum of the curve is at 1.4. Curves (b) use the constant 1.4 ratio from this work. Right: Absolute sedimentation times in $2 \mathrm{~cm}$ high water column, calculated for spherical gold particles. $T=277.15 \mathrm{~K}$ was used. Small values of $\alpha$ (left) correspond to large particle diameters (right).

We note that our numerical solver enables full simulation of the sedimentation process. This can be used to study in more detail the evolution of the concentration gradient and the attainment of equilibrium. A different metric 
for the distance to equilibrium may be used instead of the difference between the two outermost points, such as the root-mean-square deviation over the entire curve, or a metric that is representative to the particular measurement used.

We performed numerical simulations of the attainment of sedimentation equilibrium for different values of $\alpha$. The numerical solver allows us to study also the case of $\alpha<0.1$ not covered by Equation (S17). Figure S6, shows how the 'distance to equilibrium' $\epsilon$ (Eqn. S16) evolves over time (relative to the sedimentation time $\left.t_{\text {sed }}\right)$. For all values of $\alpha$, equilibration is near completion at $t \approx 1.4 t_{\text {sed }}$, even for the 'worst case' $\alpha=0.1$. As predicted by Eqn. (S17), equilibrium is attained relatively faster $\left(t<1.4 t_{\text {sed }}\right)$ for larger $\alpha$ (smaller particles).

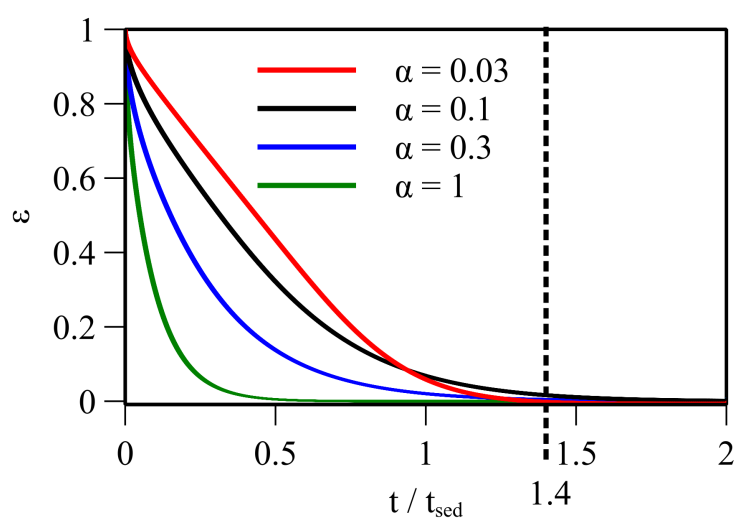

Figure S6 - Approach to sedimentation equilibrium, obtained using the numerical Mason-Weaver solver, for different values of $\alpha$. Equilibration is always virtually complete at $t=1.4 t_{\text {sed }}$. For large $\alpha$, time to equilibrium becomes shorter.

This brief analysis shows that $t_{\text {equil }} \approx 1.4 t_{\text {sed }}$ is useful as a rough approximation. It never underestimates the time to equilibrium. However, it does overestimate the time to equilibrium for the smaller particles, in which case Equation (S22) becomes relevant. 


\section{S.5 Experimental test of the centrifugation parame- ters recommmended by the Mason-Weaver model}

a.

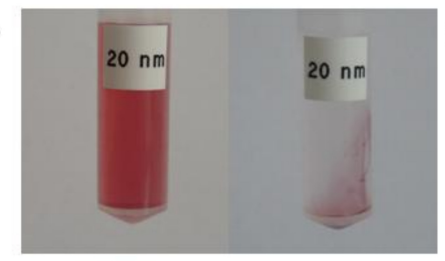

b.

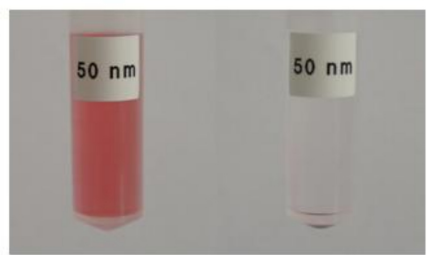

c.

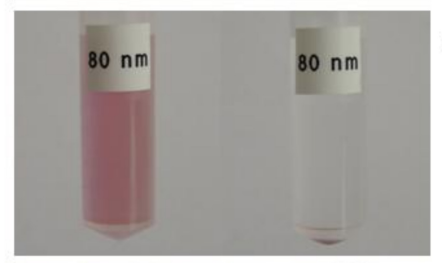

d.

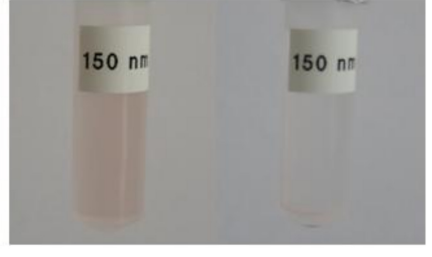

a.

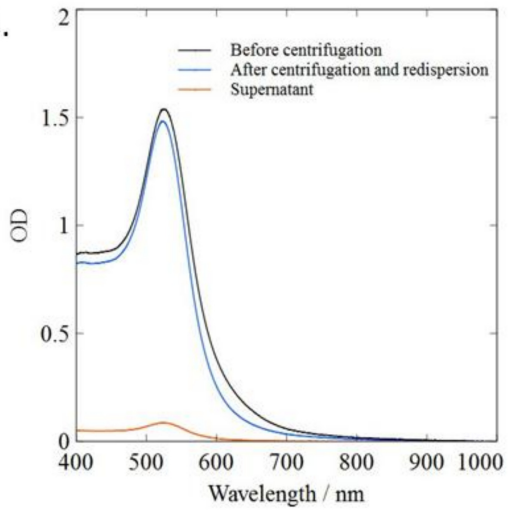

c.

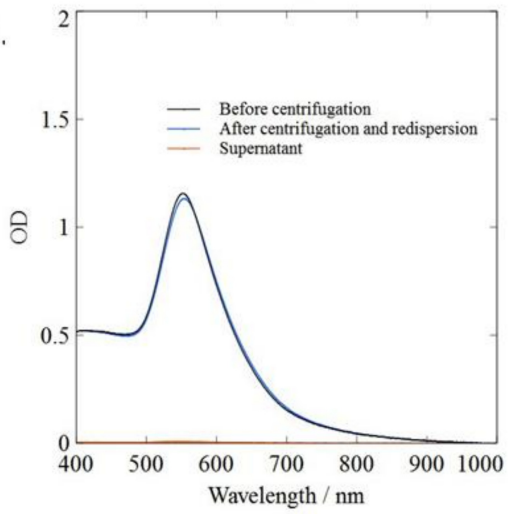

b.

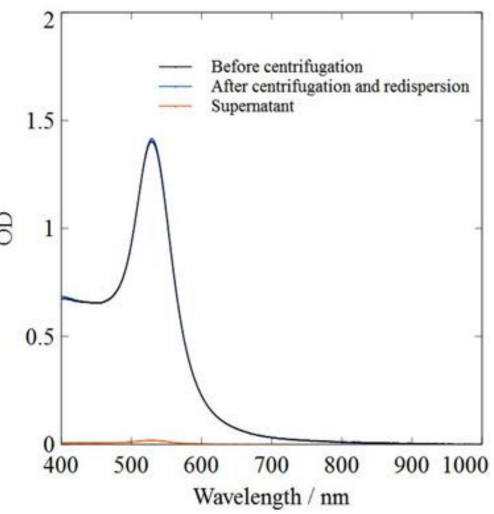

d.

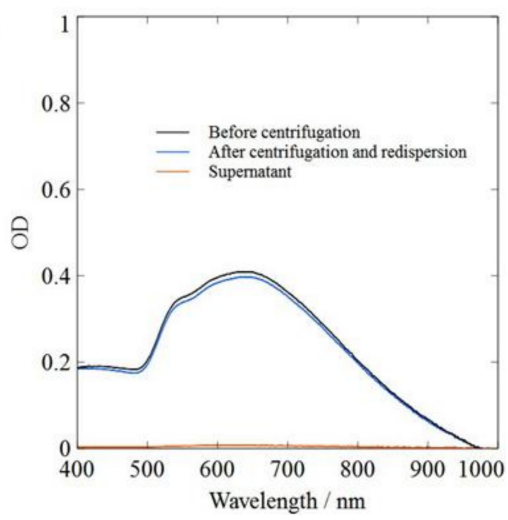

Figure S7 - Centrifugation of gold nanospheres in water, for 30 min. at the centrifugal acceleration recommended by the Mason-Weaver model. Diameters: (a) $20 \mathrm{~nm}$, (b) $50 \mathrm{~nm}$, (c) $80 \mathrm{~nm}$, (d) $150 \mathrm{~nm}$. Photographs on the left: centrifuge vials before and after centrifugation. Relative centrifugal force: (a) $4931 \times g$, (b) $789 \times g$, (c) $308 \times g$, (d) $88 \times g$. UV-visible extinction spectra on the right: sample before centrifugation (black) and after (blue) re-dispersal of the liquid pellet ( $5 \%$ of the initial volume); red: supernatant (95\% of the initial volume). 
a.

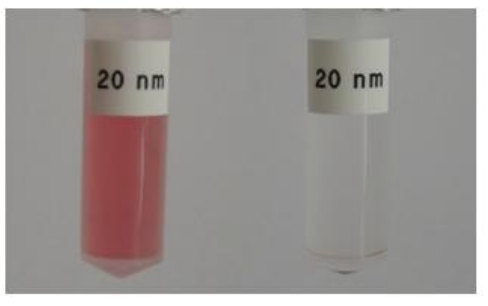

b.

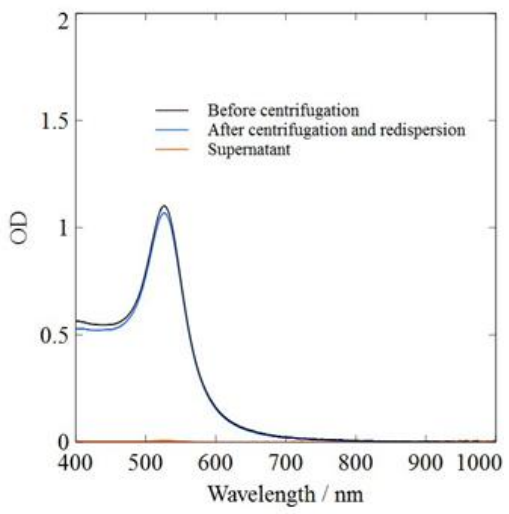

Figure S8 - Centrifugation of $20 \mathrm{~nm}$ diameter gold nanospheres in water, for 30 min., at $6000 \times$ g, i.e. $20 \%$ higher than the Mason-Weaver recommendation. 